\title{
Selective Neuronal Activation by Cochlear Implant Stimulation in Auditory Cortex of Awake Primate
}

\author{
날e A. Johnson, ${ }^{1}$ Charles C. Della Santina, ${ }^{1,2}$ and $๑$ Xiaoqin Wang ${ }^{1}$ \\ Departments of ${ }^{1}$ Biomedical Engineering and ${ }^{2}$ Otolaryngology-Head and Neck Surgery, Johns Hopkins University School of Medicine, Baltimore, Maryland \\ 21025
}

Despite the success of cochlear implants (CIs) in human populations, most users perform poorly in noisy environments and music and tonal language perception. How CI devices engage the brain at the single neuron level has remained largely unknown, in particular in the primate brain. By comparing neuronal responses with acoustic and CI stimulation in marmoset monkeys unilaterally implanted with a CI electrode array, we discovered that $\mathrm{CI}$ stimulation was surprisingly ineffective at activating many neurons in auditory cortex, particularly in the hemisphere ipsilateral to the CI. Further analyses revealed that the CI-nonresponsive neurons were narrowly tuned to frequency and sound level when probed with acoustic stimuli; such neurons likely play a role in perceptual behaviors requiring fine frequency and level discrimination, tasks that $\mathrm{CI}$ users find especially challenging. These findings suggest potential deficits in central auditory processing of CI stimulation and provide important insights into factors responsible for poor CI user performance in a wide range of perceptual tasks.

Key words: auditory cortex; cochlear implant; marmoset

\section{Significance Statement}

The cochlear implant (CI) is the most successful neural prosthetic device to date and has restored hearing in hundreds of thousands of deaf individuals worldwide. However, despite its huge successes, CI users still face many perceptual limitations, and the brain mechanisms involved in hearing through CI devices remain poorly understood. By directly comparing single-neuron responses to acoustic and CI stimulation in auditory cortex of awake marmoset monkeys, we discovered that neurons unresponsive to CI stimulation were sharply tuned to frequency and sound level. Our results point out a major deficit in central auditory processing of $\mathrm{CI}$ stimulation and provide important insights into mechanisms underlying the poor $\mathrm{CI}$ user performance in a wide range of perceptual tasks.

\section{Introduction}

An enormously successful neural prosthesis is the cochlear implant $(\mathrm{CI})$, which has restored hearing sensation to $>300,000$ individuals with profound hearing loss (Wilson and Dorman,

Received May 27, 2016; revised 0ct. 5, 2016; accepted 0ct. 10, 2016.

Author contributions: L.A.J. and X.W. designed research; L.A.J. and C.C.D.S. performed research; L.A.J. analyzed data; L.A.J., C.C.D.S., and X.W. wrote the paper.

This work was supported by National Institutes of Health National Institute on Deafness and Other Communication Disorders Grat R01DC014503 to X.W., Grant F31DC010321 to L.A.J., and Grant P30 DC005211 to the Center for Hearing and Balance at Johns Hopkins University, and a grant from the Kleberg Foundation to X.W. We thank J. Estes and N. Sotuyo for help with animal care; P. Loizou and D. Kim (University of Texas Dallas) for providing the cochlear implant stimulator current source; and Z. Smith (Cochlear Limited) for assistance obtaining implant electrodes.

The authors declare no competing financial interests.

Correspondence should be addressed to Dr. Xiaogin Wang, Department of Biomedical Engineering, Johns Hopkins University School of Medicine, 720 Rutland Avenue, Traylor 410, Baltimore, MD 21025. E-mail: xiaoqin.wang@jhu.edu.

L.A. Johnson's present address: Department of Neurology, University of Minnesota, 20016 th Street SE, Minneapolis, MN 55455

DOI:10.1523/JNEUROSCI.1699-16.2016

Copyright $\odot 2016$ the authors $\quad 0270-6474 / 16 / 3612468-17 \$ 15.00 / 0$
2008; Shannon, 2012). Despite this clinical success, CI users still face many perceptual limitations. A consistent but unexplained finding in CI research is the large variability in performance among CI users even when etiology, duration of deafness, electrode placement, and age of implantation are taken into account (Pyman et al., 2000; Blamey et al., 2013). Many CI users have difficulty in recognizing melodies in music (McDermott, 2004), and struggle to understand speech in noisy environments (Friesen et al., 2001; Stickney et al., 2004). While normal hearing listeners can perceive sound intensity changes over a $120 \mathrm{~dB} d \mathrm{dy}-$ namic range (Moore, 2012), the difference between audible thresholds and uncomfortable loudness in electric hearing via CI is considerably more constrained, at a range of 3-20 dB (Zeng et al., 2002; Moore, 2003). Although such perceptual deficits likely have peripheral causes, our understanding of how they impact the central auditory system is limited. The success of a CI relies on the ability of the central auditory system to adequately resolve and process electric stimuli (Middlebrooks et al., 2005; Moore and Shannon, 2009). The observation that CI performance can 
improve markedly over time after the device is activated also attests to the importance of plasticity and perceptual learning (Wilson et al., 2011; Blamey et al., 2013).

To understand how CI input is perceived, one must understand how the brain encodes and responds to CI stimulation. With few exceptions (Wang et al., 1999; Kirby and Middlebrooks, 2012), previous CI animal physiology studies have been commonly conducted under anesthesia. Studies in both acoustic hearing (Wang et al., 2005) and electrical hearing (Chung et al., 2014) animals have reported marked differences in neural responses between awake and anesthetized preparations. We have developed a new CI model based on a highly vocal nonhuman primate, the common marmoset (Callithrix jacchus), to overcome these obstacles (Johnson et al., 2012). Marmosets have a similar hearing range $(125 \mathrm{~Hz}$ to $36 \mathrm{kHz}$, Osmanski and Wang (2011)) and organization of auditory cortex (de la Mothe et al., 2006) as humans and has been extensively studied in our laboratory in the past two decades (Bendor and Wang, 2005; Wang et al., 2005; Wang, 2007). By implanting a multicontact CI electrode array in one ear and leaving the other ear acoustically intact, we were able to directly compare responses to acoustic and CI stimulation on the basis of single neurons in the primary auditory cortex (A1) of awake marmosets. This unique approach allowed us to assess each neuron's responses to both acoustic and CI stimulation, delivered separately or in combination, at a detailed level that is not feasible in other preparations. An interesting and surprising observation of this study was that, whereas most A1 neurons in awake marmosets responded to appropriately chosen acoustic stimuli (Wang et al., 2005), many did not respond to CI stimulation, especially in the hemisphere ipsilateral to the CI implant. By analyzing each neuron's acoustic and electrical response properties, we were able to elucidate functional properties of A1 neurons that explain the activation by CI stimulation.

Last, one development that makes this present study especially interesting and timely is that recent years have witnessed a rapid increase in the popularity of single-side cochlear implantation in individuals with single-sided deafness (Tokita et al., 2014), a situation similar to the unilaterally implanted animals in our study. Our results comprise an unprecedented view into cortical neuronal activity during unilateral electrical cochlear stimulation in subjects with normal contralateral hearing.

\section{Materials and Methods}

Unilateral cochlear implantation. All materials and methods were approved by the Johns Hopkins University Animal Use and Care Committee. Four adult (three male, one female) marmoset monkeys (Callithrix jacchus) with previously normal hearing were implanted with an 8 -channel CI electrode (Cochlear) unilaterally in the right cochlea. The CI-implanted ear was treated with neomycin in 2 of 4 animals. To facilitate the characterization of the acoustic response characteristics of A1 neurons, the left ear was kept intact in all animals.

Details of this electrode array, marmoset cochlear anatomy, and surgical approach are described in a previous report (Johnson et al., 2012), which determined that this electrode array can be inserted up to $8 \mathrm{~mm}$ in the marmoset cochlea, covering an estimated frequency-place range of $\sim 3-20 \mathrm{kHz}$. Marmosets have a normal hearing range of $0.125-36 \mathrm{kHz}$ (Osmanski and Wang, 2011) and primary vocal range of $5-9 \mathrm{kHz}$ (Pistorio et al., 2006). The CI surgery was conducted concurrently with the head-post implantation, which is described in detail in a previous study (Lu et al., 2001). The CI electrode array was inserted through a cochleostomy centered $\sim 1 \mathrm{~mm}$ anteroinferior to the round window. The electrode lead wire and connector plug were imbedded in the head cap and connected to a custom current stimulator during experimental sessions. In 2 animals (M57U and M77W), the CI electrode was inserted into an otherwise intact cochlea. In the other 2 animals (M5X and M3Y), the cochlea was deafened just before electrode insertion with multiple intrascalar injections of neomycin sulfate ( $10 \%$ solution), which is toxic to hair cells (Nuttall et al., 1977). Perilymph was gently removed by suction at the cochleostomy margin, and the cochlea was filled with neomycin solution using a flexible syringe inserted through the cochleostomy. After 1-2 min, the fluid in the cochlea was removed by suction and filled again with neomycin solution. This process was repeated four times. To further reduce the likelihood of residual auditory input in the CI ear in these 2 animals, the middle ear ossicles of the implanted ear were disarticulated and the incus was removed.

Assessment of residual hearing. To assess residual hearing in the implanted ear, we measured responses to rectangular acoustic clicks $(0.1 \mathrm{~ms}$ duration) using differential recordings from the most apical and basal CI electrode contacts. An extra-cochlear contact in the neck served as ground. Stimuli were presented at various sound levels from a free-field speaker $1 \mathrm{~m}$ in front of the animal, as used during cortical recording sessions. Signals were amplified $\times 10,000$ and bandpass filtered $100-$ $3000 \mathrm{~Hz}$. These signals, referred to here as auditory brainstem response (ABR), can potentially include (in order of latency): cochlear microphonic and auditory nerve compound action potential (from the implanted ear), as well as brainstem responses (from either implanted or nonimplanted ear). At high sound levels ( $\geq 60 \mathrm{~dB}$ SPL), animals not treated with intrascalar injections of neomycin sulfate had prominent short latency responses $<2.5 \mathrm{~ms}$, likely reflecting the cochlear microphonic and auditory nerve compound action potentials and some degree of residual hearing. High thresholds for response were likely due to electrode insertion trauma and cochlear hair cell loss (Kennedy, 1987; Ni et al., 1992). In all animals, the implanted ear was occluded with ear impression material during neural recording sessions. This occlusion of the implanted ear effectively abolished the short latency auditory response in animals not treated with neomycin. In neomycin-treated animals, ABRs showed no early response $(<2.5 \mathrm{~ms})$ up to $90 \mathrm{~dB}$ SPL regardless of ear occlusion. The highest sound level typically used in experimental sessions was $80 \mathrm{~dB}$ SPL. With deafness confirmed in 2 animals and ear occlusion used in all animals, we contend that our recordings largely reflect neural responses to monaural acoustic (left ear) and monaural CI (right ear) stimulation.

Electrophysiology recordings. Neural recording sessions were performed in a double-walled, soundproof chamber (Industrial Acoustic) with an interior covered by 3 -inch acoustic absorption foam (Sonex). Daily sessions typically lasted $3-5 \mathrm{~h}$ and continued many months after implantation (see Fig. $3 B$ ). Animals were awake and semirestrained in a custom primate chair and closely monitored by video during recordings. Single units were recorded in primary auditory cortex, alternating every 1-2 weeks between hemispheres. Units were recorded using tungsten microelectrodes (2-5 M $\Omega$, A-M Systems) and isolated online using a template based spike discriminator (MSD, Alpha Omega Engineering). Exact laminar position of our recordings was not determined. However, we recorded neurons as they were encountered by electrode penetrations perpendicular to the cortical surface; and because the electrode was advanced slowly and extensive effort was made to drive neuronal firing in each neuron, sampling was likely biased to neurons in upper cortical layers $2 / 3$. After each unit was isolated, its responsiveness to acoustic and CI stimulation was respectively tested.

Each CI stimulation pulse produced a large electrical artifact in the recording signal $400-500 \mu$ s in duration, creating a potential for data loss if spikes occurred concurrently. At low stimulus rates, the data loss was minimal due to the small number of stimulus pulses and the time delay between peripheral stimulation and cortical response. However, the possible number of spikes occluded by artifact increased with stimulation rate, so for rapid pulse rates $(\geq 100 \mathrm{~Hz})$, we implemented a digital comb filter (iircomb, filtfilt in MATLAB, The MathWorks) with spectral nulls at integer multiples of the stimulation rate. This effectively removed electrical artifact with only modest reduction in unit waveform size. Responses to rapid pulse train CI stimuli were then sorted offline using template based spike sorting similar to the online sorting methods.

Acoustic and CI stimuli. Acoustic stimuli were generated digitally (Tucker Davis Technologies) and delivered through a free-field speaker $1 \mathrm{~m}$ in front of the animal. Frequency tuning curves and rate-level func- 
tions were determined using tones or bandpass noise ( $\leq 1$ octave), $100-$ $200 \mathrm{~ms}$ in duration with interstimulus intervals $\geq 500 \mathrm{~ms}$. The frequency axis was typically sampled in $0.1-0.2$ octave steps over a range of 2-4 octaves. In some cases, a smaller octave spacing and range were used for neurons responding to a very narrow range of frequencies (see Fig. 6D). The sound level axis was sampled every $10 \mathrm{~dB}$ for rate level functions over a range of -10 to $80 \mathrm{~dB}$ SPL. In a subset of neurons, frequency response areas (FRAs) were assessed by measuring frequency tuning curves across multiple sound levels in $20 \mathrm{~dB}$ steps. Tone stimulus durations for FRAs ranged from 50 to $200 \mathrm{~ms}$ depending on neuron response characteristics. For example, neurons with brief onset responses (see Fig. 6A) were presented with 50 or $100 \mathrm{~ms}$ tones, whereas neurons with longer latency and/or sustained responses were delivered longer, $200 \mathrm{~ms}$ tones. Twotone stimuli consisted of one tone at the unit's best frequency presented concurrently with another tone spanning the same range of frequencies used in generating the frequency tuning curve. Two-tone stimuli were presented at the same sound level or within $10 \mathrm{~dB}$ of the sound level used for the frequency tuning curve, which was typically 20-30 dB above neural threshold.

CI stimuli were generated digitally at $2.5 \mathrm{MHz}$ sampling rate and delivered to a custom current stimulator through a digital I/O PCI board (National Instruments). The 8-channel bipolar stimulator used in these studies was developed by Dr. Philip Loizou's CI Laboratory at University of Texas Dallas under an NIDCD contract (Kim et al., 2009). Electrically isolated, charge balanced biphasic current pulses ( $100 \mu$ s per phase, $50 \mu \mathrm{s}$ interphase gap) were delivered to adjacent intracochlear electrode contacts (more apical electrode initially cathodic). Electrode contacts are numbered 9 (most apical) through 2 (most basal) (see Fig. 1B). We refer to the pair of electrode contacts current is passed through as a CI electrode (e.g., electrode $9-8$ is the most apical pair of contacts, electrode 3-2 the most basal). A subset of neurons was tested with monopolar stimulation, with current delivered to an intracochlear contact and distant silver ball electrode chronically implanted in the neck. After surgery recovery, electric auditory brainstem responses were measured to confirm effectiveness of electric CI stimulation to activate the auditory pathway. When neural recordings began in each animal, the current levels used were initially based on the levels used in the electric auditory brainstem responses, but then adjusted based on animal tolerance and cortical neural responses. In one animal, M3Y, electrode 3-2 had much higher impedance than other electrodes and was not used.

The CI electrode with the largest firing rate response that was significantly higher than the neuron's spontaneous firing rate was defined as the best electrode. Extensive effort was made to identify the CI stimulation parameters (i.e., electrode, current level, repetition rate) that could drive neuronal firing. Our goal when choosing the CI stimulation parameters was to evaluate how one could drive A1 neurons using single-channel CI stimulation. Given what we know about the neural selectivity and nonlinearity in auditory cortex of awake marmosets (e.g., Sadagopan and Wang, 2009), and considering that cortical single neuron responses to CI stimulation in awake primates have not been described previously, we did not take for granted which parameter combination would be effective. We therefore explored a wide range of current levels (in $10 \mu \mathrm{A}$ steps ranging from zero to within animal tolerance, typically maximum levels of $150-170 \mu \mathrm{A})$ and repetition rates $(4-488 \mathrm{~Hz}$ with near octave spacing, example shown in Fig. 9A). For the purposes of this study, if a neuron responded significantly above spontaneous firing rate to any of the CI stimuli presented, it was considered to be "CI-driven." Because the time to hold a neuron varied from neuron to neuron and there was a large parameter space to examine, not every stimulus combination was delivered. However, a standard stimulus set was identified for each animal, so that, at the very least, responses to a common set of stimuli, presented to all CI tested neurons in a given animal, could be assessed. This common stimulus set consisted of the following: three current levels (130, 90, and $60 \mu \mathrm{A}$ for M57U, M77W, and M5X, and 160, 110, and $70 \mu \mathrm{A}$ for M3Y), delivered at each electrode, using three repetition rates (single pulses, as well as $200 \mathrm{~ms}$ duration pulse trains delivered at $32 \mathrm{~Hz}$ and $257 \mathrm{~Hz}$ ). Based on initial recordings, we were able to get a sense of the dynamic range of CI-driven neurons, and the three current levels indicated above were chosen to capture that dynamic range (spanning levels from near threshold to suprathreshold but within animal tolerance). The repetition rates were chosen based on our initial observations that some neurons responded preferentially to CI stimuli delivered at rapid repetition rates, whereas other neurons responded best to slower modulations. Each neuron was typically tested at many additional current levels and repetition rates beyond the standard stimulus set.

$\mathrm{CI}$ and acoustic binaural combination stimuli consisted of the best frequency (BF) acoustic stimulus presented concurrently with a $200 \mathrm{~ms}$ duration, $257 \mathrm{~Hz} \mathrm{CI}$ stimulus delivered sequentially to each electrode at a high current level (see Fig. 8A). CI and acoustic binaural rate-level functions (see Fig. $6 C$, bottom right) consisted of the BF acoustic stimulus presented concurrently with the CI stimulus delivered at a range of current levels (10-20 $\mu \mathrm{A}$ steps) to the electrode found to have greatest suppressive effect.

Data analysis. Data were analyzed using custom software written in MATLAB. A neuron response was defined as stimulus-driven if it had significant discharge rate relative to spontaneous rate (Wilcoxon ranksum, $p<0.05)$ and a discharge rate of at least 5 spikes/s. Responses to acoustic stimuli were analyzed in a window $10 \mathrm{~ms}$ after stimulus onset to $20 \mathrm{~ms}$ after stimulus offset. Responses to CI stimuli were analyzed $10 \mathrm{~ms}$ after stimulus onset to $50 \mathrm{~ms}$ after stimulus offset. Spontaneous firing was typically measured in a $200 \mathrm{~ms}$ window prestimulus onset. The mean spontaneous rate was subtracted from the mean driven rate in all analysis unless otherwise noted.

The stimulus frequency with the highest stimulus-driven firing rate response was defined as the BF. Frequency tuning curves were created and analyzed similar to Sadagopan and Wang (2008). Responses were thresholded at $20 \%$ of peak response, interpolated to 40 steps/octave, and smoothed with a 5-point moving window. We then fit an areamatched rectangle to the tuning curve, fixing its position at the centroid of the tuning curve and height at maximum firing rate. The rectangle width was taken as the measure of bandwidth. This bandwidth measure approximates the FWHM of a Gaussian, but has the advantage of not assuming any particular tuning curve shape. The quality factor $(\mathrm{Q})$ was defined as the $\mathrm{BF}$ of a unit divided by its bandwidth. In a subset of neurons, frequency tuning curves were obtained at multiple sound levels to create FRAs. Bandwidth measures from the FRA were used to calculate a shape index (SI), defined as the bandwidth at the loudest sound level divided by the bandwidth at the sound level that evoked maximum response. V-shaped neurons had bandwidths that increased with increasing level and therefore SI values $\geq 1$. O-shaped neurons had bandwidths that decreased with level and SI values near zero. To further assess a unit's selectivity to frequency and level, an FRA response area was calculated by summing the number of points in the tuning curve at each level with discharge rates $\geq 2$ SDs above mean spontaneous rate. Whereas the SI indicates the shape of the FRA, the response area measure gives an indication of the size of the significant driven activity in the FRA.

A monotonicity index (MI) was defined as the mean discharge rate at the highest sound level tested (typically $80 \mathrm{~dB}$ SPL) divided by the mean discharge rate at the unit's best sound level. Spontaneous rate is subtracted before this calculation, so negative MIs reflect suppressed responses at the highest sound level. Median Q values and MIs of CI-driven and CI-nonresponsive populations were compared using a Wilcoxon rank-sum test to determine statistical significance $(p<0.05)$. Cumulative distribution functions were compared using a Kolmogorov-Smirnov test $(p<0.05)$.

The bandwidth index (BI) reflects the unit's preference for broadband or narrowband acoustic signals. Bandpass noise stimuli used for calculating BI were centered at the unit's BF and ranged from 0 (tone) to 2 octaves wide in 0.2 octave steps. Mean discharge rates were normalized by the maximum mean rate, and the $\mathrm{BI}$ was defined as the maximum rate evoked by the two broadest stimuli used (typically 1.8 and 2 octave bandpass noise) minus the maximum rate evoked by the two most narrow stimuli (pure tone and narrowband noise, typically 0.2 octave bandpass noise). Spontaneous rate was not subtracted for this calculation. Many neurons responded only to narrowband stimuli and therefore had BI values near -1 . A BI value near 0 indicates that the unit responded equally well to narrow and broadband stimuli. 
A
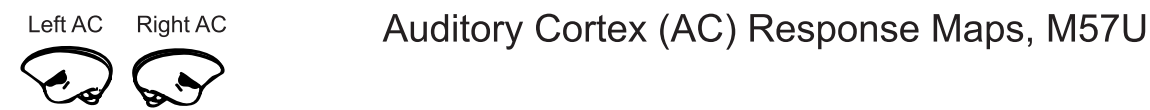

(1) (2) 29

Best Frequency (Acoustic Stimulation)

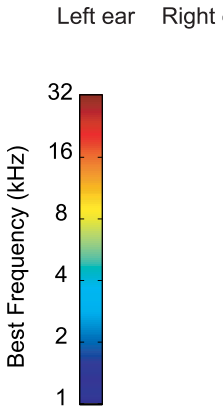

\section{ear}

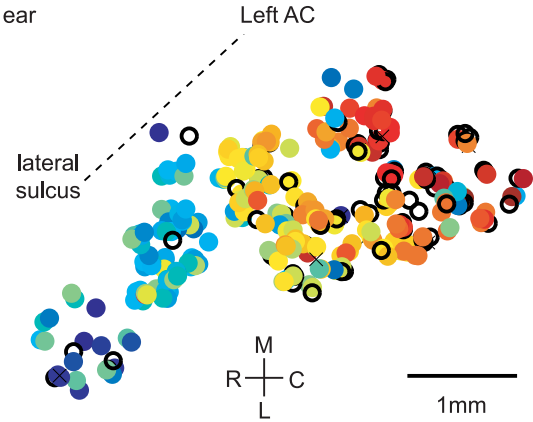

$\times$ Did not test

O Non-responsive (NR)

\section{B}

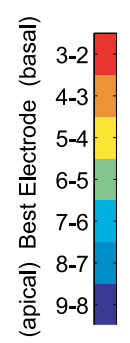

$\times$ Did not test

O Non-responsive (NR)

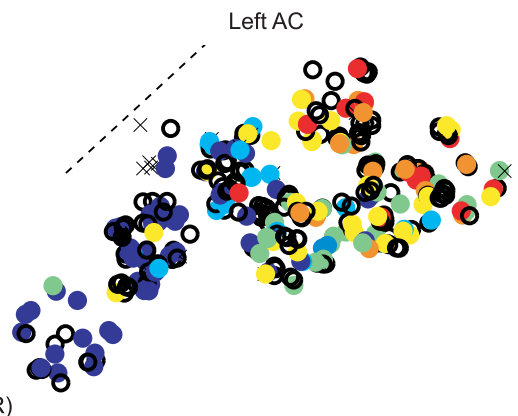

Best Electrode (Cochlear Implant Stimulation)

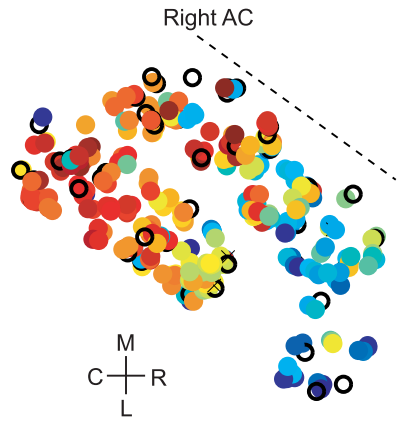

C Best Frequency vs Best Electrode

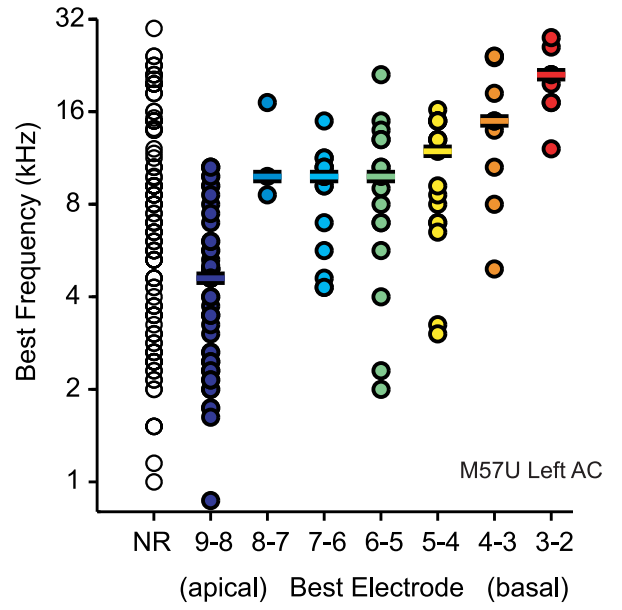

\section{D}

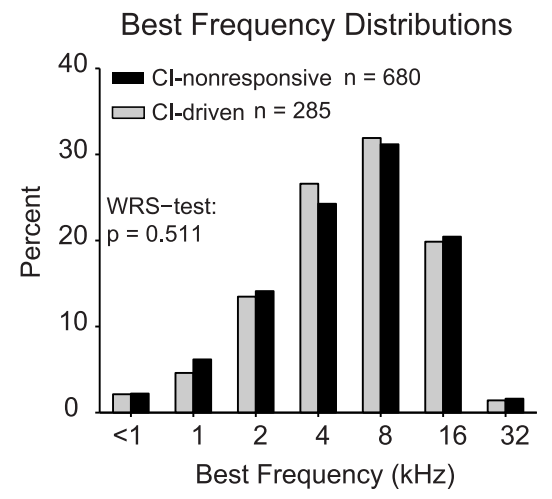

Figure 1. Topographic maps of auditory cortex responses to acoustic and Cl stimulation. A, Acoustic BF maps of left and right auditory cortex (AC) of a marmoset implanted unilaterally in the right cochlea with a Cl electrode array (inset plot). Dashed lines indicate approximate positions of the lateral sulcus. Each circle represents a single neuron recorded at that cortical surface location, color-coded by its BF. Black open circles represent neurons nonresponsive (NR) to acoustic tones and bandpass noise. Crosses indicate neurons only tested with $\mathrm{Cl}$. $\boldsymbol{B}$, $\mathrm{Cl}$ best electrode maps show the same neurons as in $A$, with color corresponding to $\mathrm{Cl}$ best electrode. Stimulation was between adjacent contacts in the electrode array, indicated by the pair of numbers on the $y$-axis. Symbols are the same as in $A$. Crosses indicate neurons only tested with acoustic stimulation. $C$, BF versus best electrode comparison of neurons from the left auditory cortex of one animal (M57U), contralateral to Cl stimulation. Horizontal bars represent median BF values for each best electrode. The first column represents the BFs of Cl-nonresponsive neurons. D, BF distributions for $\mathrm{Cl}$-driven and $\mathrm{Cl}$-nonresponsive neurons from all 4 animals. 
The excitation/suppression (E/S) index reflects the degree of two-tone suppression relative to excitation as measured from the tone-alone frequency tuning curve. Frequencies spanning 1.5 octaves about the unit's $\mathrm{BF}$ were used in this calculation. The frequency tuning curve was normalized by the BF response, and the area under the curve was defined as the excitation area. To compute the suppression area, first the BF response was subtracted from the two-tone responses, and the resulting two-tone frequency tuning curve was normalized by the BF response. To focus on substantial suppression, values $\geq-0.3$ (reflecting a reduction in discharge rate $<30 \%$ pure tone BF response) were set to zero (Kadia and Wang, 2003), and the area under the curve was defined as the suppression area. The E/S index was defined as follows: (excitation area - suppression area)/(excitation area + suppression area). An E/S index of 1 reflects no two-tone suppression. Negative values reflect larger suppression areas than excitation areas.

Neurons tested with CI and Acoustic binaural combination stimuli ( $n=192$ ) were defined as CI-suppressed if at least one stimulation channel or stimulation level significantly reduced the discharge rate relative to the acoustic alone condition (Wilcoxon rank-sum test, $p<0.05$ ) by at least $50 \%$. Neurons that did not meet this criterion were defined as CI-no effect. Neurons showing significant CI facilitation $(n=9)$ were not included in subsequent analysis. Statistical analyses were performed using MATLAB using nonparametric tests (e.g., Wilcoxon signed-rank test) given the non-normal distribution of most variables studied.

\section{Results}

This report is based on 1408 well-isolated single neurons recorded in awake condition from the primary auditory cortex (A1) of eight hemispheres in four marmosets that were unilaterally implanted with CI electrodes in the right ear (see Materials and Methods). Each single neuron was carefully isolated online and individually studied using the chronic single-unit recording technique our laboratory has developed and refined for marmosets (Wang et al., 2005). Of 1408 recorded neurons, 1374 neurons were tested with acoustic stimuli (1048 were significantly driven) and 1306 neurons were tested with CI stimuli (355 were significantly driven). We were able to hold most neurons long enough to test both stimulus modalities (1272 of $1408, \sim 90 \%)$. A1 in both left and right hemispheres was studied in each animal. This permitted both single-neuron and population analyses of cortical responses to contralateral and ipsilateral stimulation for both acoustic and CI stimuli. In this study, the electrical stimulation of the cochlea, referred to as CI stimulation hereafter, was delivered to one of seven electrode pairs of a CI electrode array. CI stimulation occurred only during experimental sessions. The effects of chronic CI stimulation will be evaluated in future studies.

We performed this study in unilaterally CI-implanted marmosets because of an important concern in previous studies of deaf or deafened animals, namely, how would the precise location of a particular field in auditory cortex (e.g., A1) be determined and whether an auditory neuron is being studied in a completely deaf animal? This is less a concern if one studies the auditory nerve or some subcortical auditory structures where anatomical landmarks can better guide your recording locations. In cortex (particularly for an animal like marmoset with a flat cortex), there are no clear anatomical landmarks to indicate where exactly A1 is located on the cortical surface and to distinguish A1 from neighboring auditory fields. We believe knowing what kinds of neurons are being studied and where the neurons are located in a particular cortical field is crucial for understanding the effectiveness of CI stimulation and ultimately for improving CI stimulation paradigms. By leaving one ear acoustically intact and taking advantage of binaural response properties of A1 neurons, we were able to precisely locate A1 and quantitatively determine acoustic tuning properties and the selectivity of every neuron we studied with CI stimulation, which is not feasible in a totally deaf animal.

\section{Cochlear electrical stimulation is less effective in activating cortical neurons than acoustic stimulation}

We classified a neuron as acoustically driven if it had a stimulusdriven response (see Materials and Methods) to acoustic presentation of tones or bandpass noise, two types of stimuli that drive most of A1 neurons in awake marmosets, albeit not necessarily optimally (Wang et al., 2005). We also systematically tested each neuron's response to pulsatile electric stimuli delivered sequentially to individual electrode pairs in the CI electrode array, using a wide range of pulse rates and current levels (Fig. 2). Representative firing rate versus current level functions acquired in each of the 4 animals are shown in Figure 2. Most neurons responsive to $\mathrm{CI}$ stimulation either reached saturation in firing rate or were suppressed at the highest current level tested (Fig. $2 A$ ). Figure $2 B$ shows examples of neurons that were nonresponsive to CI stimulation across the range of current levels used. Considerable effort was made to seek out an effective CI stimulus (for more details, see Materials and Methods). Cortical tonotopic maps and $\mathrm{CI}$ response maps were created based on the BF (or center frequency for bandpass noise stimuli) and best CI electrode (defined as the $\mathrm{CI}$ electrode that best activated a neuron), respectively (Fig. $1 A, B)$. Each circle on these plots represents a sampled neuron, with color corresponding to its BF or best electrode. Acoustic BF maps show clear tonotopic organization in $\mathrm{A} 1$, with a high to low-frequency gradient along the caudorostral axis (Fig. 1A).

Whereas the majority of neurons in both hemispheres respond to acoustic stimuli (Fig. $1 A$ ), best CI electrode maps reveals less responsiveness to electric stimuli (Fig. 1B). Several important observations can be made from the CI response maps. First, CI stimulation did activate a substantial subpopulation of neurons spanning the tonotopic axis, as demonstrated in the left hemisphere CI response map (contralateral to the implant, Fig. $1 B$, left plot). As expected, CI stimulation at basal electrodes tended to activate neurons with higher BFs, whereas apical electrodes activated neurons with lower BFs (Fig. 1C). This is consistent with the known tonotopic organization of the cochlea and auditory cortex. However, as evidenced by the predominance of black circles in the CI response maps, many neurons did not respond to the CI stimulation, especially in the hemisphere ipsilateral to $\mathrm{CI}$ stimulation (Fig. $1 B$, right plot).

Despite the fundamental differences between CI and acoustic modalities, we made the effort to match these stimuli whenever possible. Our approach of systematically testing a neuron's responsiveness to $257 \mathrm{~Hz}$, across the seven electrode pairs in the array, at multiple current levels is analogous to our approach of testing the neuron's responsiveness to acoustic stimuli, across a range of frequencies and at multiple sound levels; $257 \mathrm{~Hz}$ was always tested, and regularly $488 \mathrm{~Hz}$ was tested as well. These pulse train stimuli would be analogous to how a pure tone processed through a clinical CI device would be delivered to a multichannel $\mathrm{CI}$ electrode array; the tone would fall into a single bandpass filter and be delivered to a single electrode or electrode pair in the array at the defined carrier rate. Although many modern speech processors use higher carrier rates, the $257 \mathrm{~Hz}$ rate we used is near the carrier rate of $250 \mathrm{~Hz}$ that is used in the SPEAK strategy that has been used widely in clinical CI speech processors (Skinner et al., 1994). In addition, we also included bandpass noise (BW $\leq 1$ octave) for characterization of acoustic responsiveness in A1 neurons. Given the large spread of current in the cochlea during CI stimulation, it is possible that bandpass noise, which activates 
A
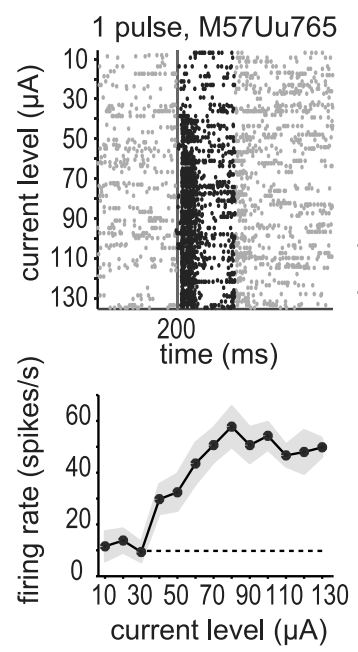

$64 \mathrm{~Hz}, \mathrm{M} 3 \mathrm{Yu} 41^{*}$
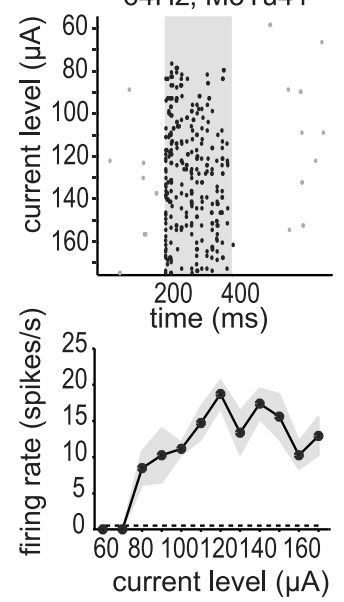

$8 \mathrm{~Hz}, \mathrm{M} 5 \mathrm{Xu} 77^{*}$
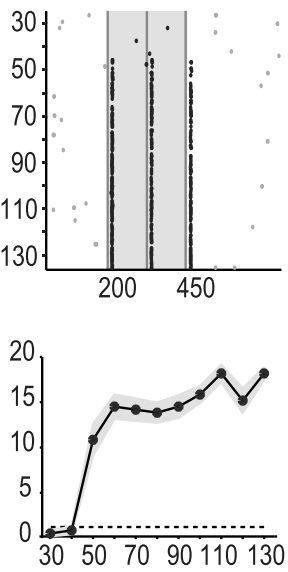

$30 \lcm{5070 \quad 90110130}$
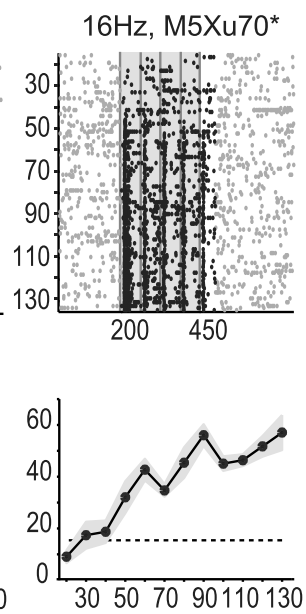

$32 \mathrm{~Hz}, \mathrm{M} 77$ Wu168
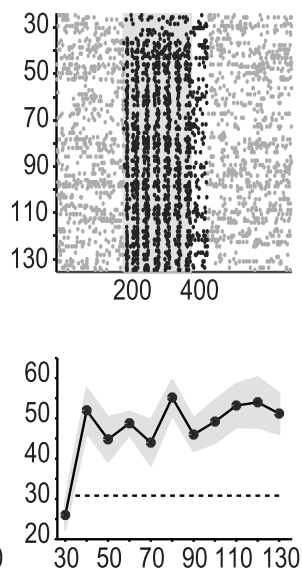

B
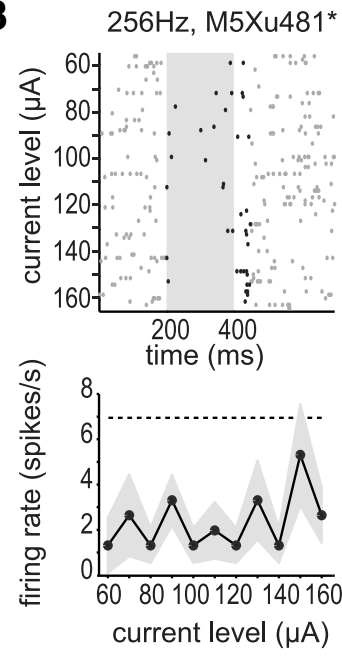

$256 \mathrm{~Hz}, \mathrm{M} 3 Y u 121^{*}$
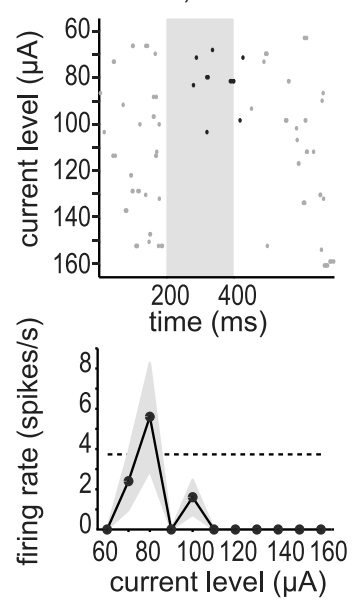

Figure 2. Representative (I stimulation rate-level functions. $\boldsymbol{A}$, Responses to electrical stimulation as a function of current level in eight $\mathrm{Cl}$-driven neurons (two from each animal). Raster plots (top) and firing rate (bottom, mean \pm SD) are shown for each neuron. Shaded region represents the period of stimulation in the raster plot. Black dots indicate calculation of firing rate. Dark vertical lines in the first three raster plots indicate times of the single, $8 \mathrm{~Hz}$, and $16 \mathrm{~Hz}$ pulse train stimuli, respectively, but are omitted for clarity in higher repetition rate stimuli. Each neuron was tested at many current levels and repetition rates across the electrode array; the rate-level functions at the neuron's best electrode and repetition rate are shown. Asterisks are placed next to unit numbers of the 2 animals that were ototoxically deafened. $\boldsymbol{B}$, Examples of $\mathrm{Cl}$-nonresponsive neurons' rate-level functions.

broader frequency place areas in the cochlea compared with pure tones, may be more analogous (though not identical) to CI stimulation. For example, noise vocoders are often used to simulate CI-processed sounds. In summary, we contend that a subset of the CI stimuli used (200 ms pulse trains delivered at $257 \mathrm{~Hz}$ ) should be considered similar to the acoustic stimuli used (100$200 \mathrm{~ms}$ pure tones and bandpass noises).

The ineffectiveness of CI stimulation compared with acoustic stimulation was observed in all 4 animals (Fig. $3 A$ ). In every animal, acoustic stimulation activated the majority of neurons $(\sim 75 \%)$ in both hemispheres. In sharp contrast, CI stimulation activated less than half of recorded neurons in the hemisphere contralateral to the $\mathrm{CI}$ (Fig. $3 \mathrm{~A}$, left) and only a small percentage of neurons $(<12 \%)$ in the hemisphere ipsilateral to the CI (Fig. $3 A$, right). Moreover, in neurons responsive to both modalities, the strength of response was significantly smaller with CI stimulation than with acoustic stimulation in both left hemisphere (A1 ipsilateral to acoustic, contralateral to CI; Fig. $4 A, n=129$, median maximum firing rate, acoustic: $34.1 \mathrm{spk} / \mathrm{s}, \mathrm{CI}: 18.2 \mathrm{spk} / \mathrm{s}, p=$ 1.81e-6, Wilcoxon rank-sum (WRS) test) and right hemisphere (A1 contralateral to acoustic, ipsilateral to CI, Fig. 4B, median maximum firing rate, acoustic: $32.5 \mathrm{spk} / \mathrm{s}, \mathrm{CI}: 19.5 \mathrm{spk} / \mathrm{s}, p=$ 0.006 , WRS test). This analysis was performed in neurons for which both acoustic and CI rate-level responses were systematically tested at multiple sound levels at BF for acoustic stimulation, and at multiple current levels at the best electrode and stimulus repetition rate for CI stimulation, respectively.

Because the electrode array was inserted only in the basal turn of the cochlea (Johnson et al., 2012), which is innervated by auditory nerve fibers tuned to higher frequencies, neurons with low BFs $(\sim<2 \mathrm{kHz})$ may not be activated by CI stimuli. However, we recorded predominately from $\mathrm{A} 1$ neurons with $\mathrm{BF}$ ranging 2-32 $\mathrm{kHz}$ (Fig. 1C). Neurons nonresponsive to CI stimulation had BFs spanning this entire range. Furthermore, when comparing the BFs of the neurons that were driven by CI stimulation and those that were nonresponsive to CI stimulation (Fig. $1 D$ ), no difference was found (median BF, CI-driven: $8 \mathrm{kHz}$, CI-nonresponsive: $8.2 \mathrm{kHz}, p=0.511$, WRS test).

Because we approached the auditory cortex perpendicularly to the cortical surface, advanced the electrode slowly, and studied neurons as they were encountered, most of the sampled neurons were recorded from more superficial layers. In Figure $4 C$, we plot 
A

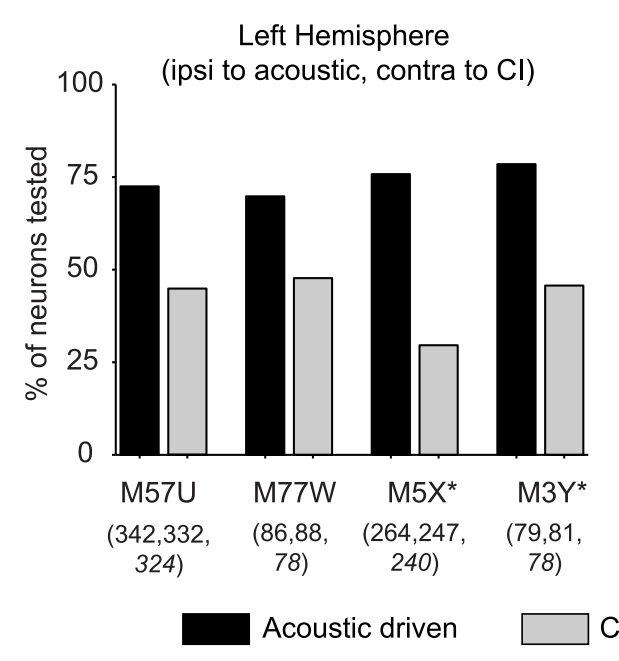

Acoustic and Cochlear Implant Responsiveness



Cl-driven \# neurons tested: (Acoustic, $\mathrm{Cl}$, both modalities)

* ototoxically deafened in implanted ear

\section{B $\quad$ Cl Responsiveness Over Time}

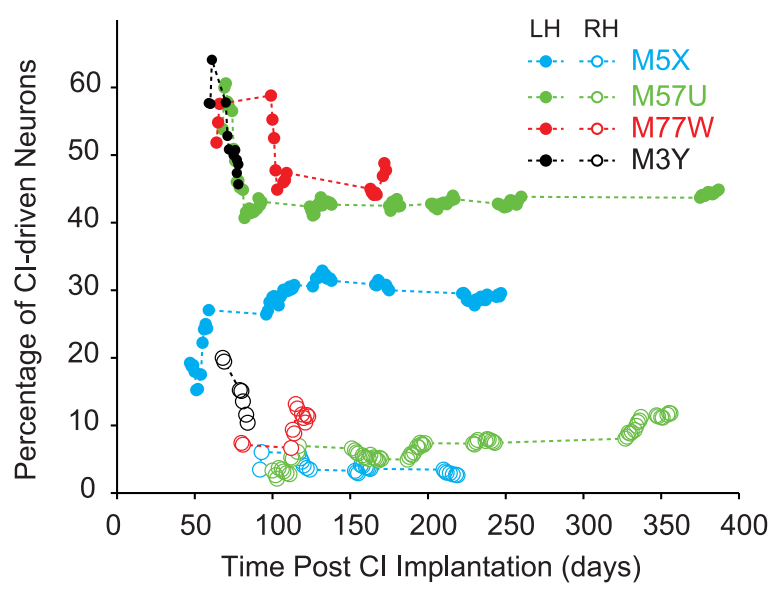

Figure 3. Comparison of acoustic and Cl responsiveness in all animals. A, Proportions of neurons that can be driven by acoustic (black bars) or Cl (gray bars) stimuli for each animal and hemisphere. Asterisks next to the animal identification numbers (M5X, M3Y) indicate that these 2 animals were ototoxically deafened immediately before Cl implantation (see Materials and Methods). The numbers in parentheses indicate the number of neurons in each animal tested with acoustic and Cl stimuli, respectively. In italics are the number of neurons tested with both modalities. The vast majority of neurons were tested with both modalities. $\boldsymbol{B}$, Proportions of Cl-driven neurons are shown for each animal and hemisphere over the time after Cl implantation, displayed as the cumulative percentage calculated at the end of an experimental session.

the distributions of recording depths of both CI-driven (dark dots) and CI-nonresponsive (gray dots) neurons. The median relative recording depths of CI-nonresponsive neurons was 0.4 $\mathrm{mm}$, whereas the median recording depth of CI-driven neurons was significantly deeper, $0.56 \mathrm{~mm}$ (WRS test, $p=9.6 \mathrm{e}-7$ ). The depth data presented here suggest that neurons in upper layers (which are involved in greater intracortical processing) are less likely to respond to CI stimulation than neurons in middle layers (which receive more thalamic inputs), implying that the paucity of CI responses observed in A1 cannot be solely attributed to subcortical processing, but possibly due to deficiencies in cortical processing of CI stimulation.

One factor that might influence our results is A1 plasticity that could affect CI responsiveness in each hemisphere over time (de Villers-Sidani and Merzenich, 2011; Fallon et al., 2014). Although effects of plasticity on neural responses were not specifically investigated in this study, recordings were made alternately in each hemisphere for up to 14 months after CI implantation. The cumulative percentages of neurons responsive to CI stimulation over time after implantation indicate that the asymmetry in CI responsiveness is evident from the start of recording (Fig. $3 B$ ), and the proportion of neurons responsive to CI stimulation remains largely unchanged throughout the duration of the experiments after an initial period. Animals used in this study did not receive continuous CI stimulation other than during the neural recording experiments. Greater neural plasticity could potentially occur in animals receiving chronic CI stimulation, a topic for future studies.

We observed similar patterns of A1 responsiveness in these two groups of animals (Fig. $3 A$ ), and we found no significant difference in the primary measures between these two groups of animals (i.e., Q values, MI, SI, BW index, E/S ratio; WRS test, $p<$ $0.05)$, so in the following Results sections data from all animals are presented together. 
A

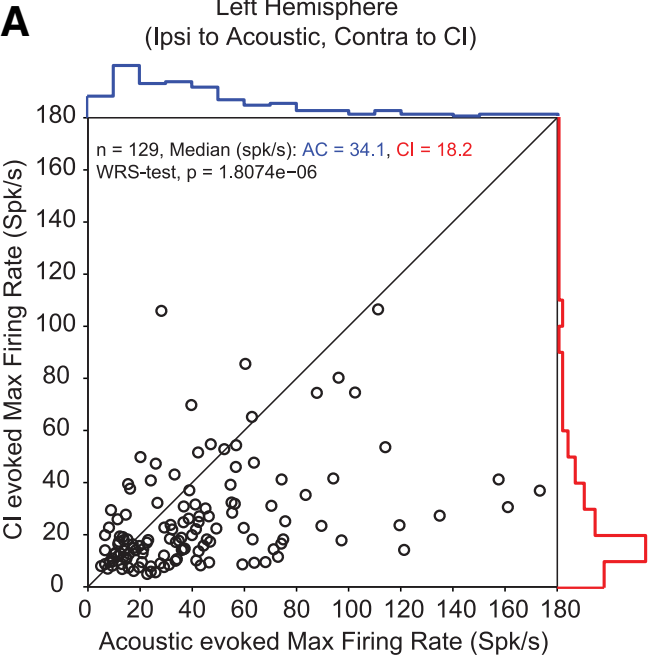

B Right Hemisphere

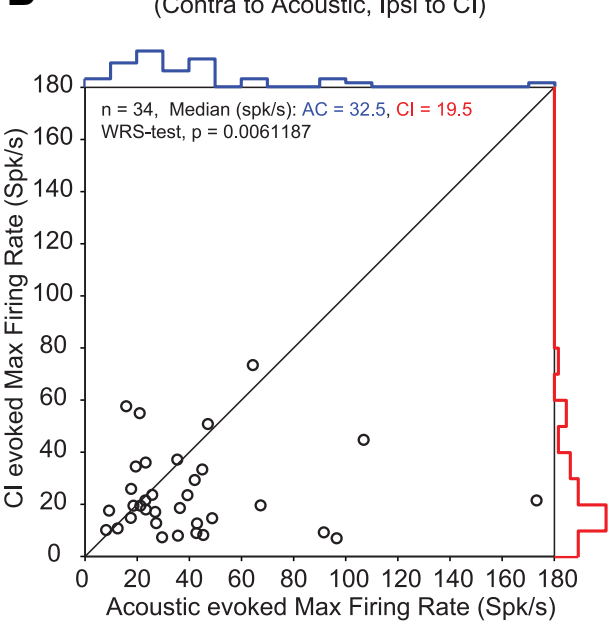

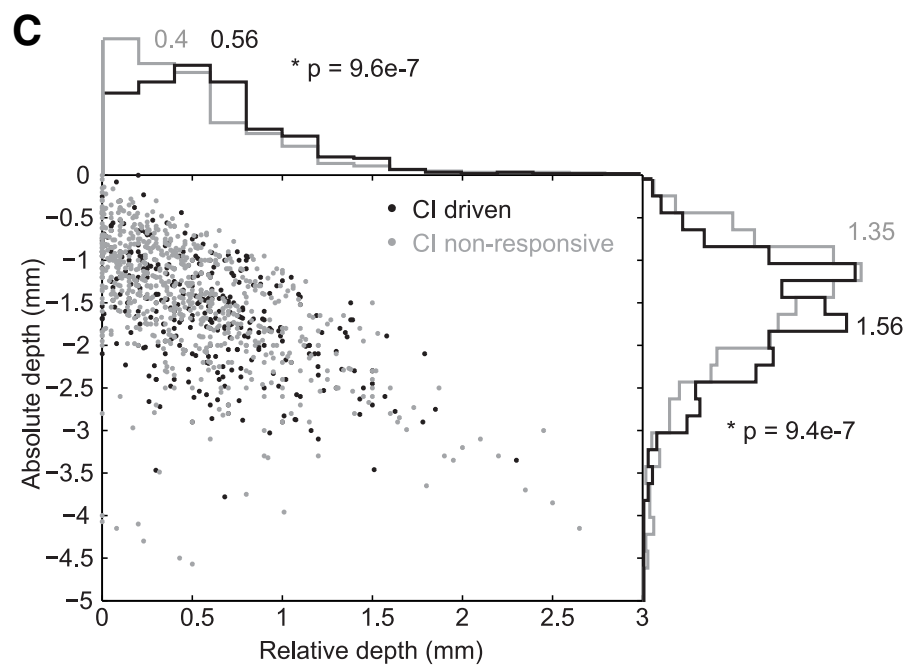

Figure 4. Comparison of strength of response in neurons responsive to both $\mathrm{Cl}$ and acoustic stimulation. Plots are based on neurons for which both acoustic and $\mathrm{Cl}$ rate-level responses were acquired (at BF for acoustic, and the best electrode and stimulus repetition rate for $\mathrm{Cl}$ ). The maximum firing rates for acoustic and $\mathrm{Cl}$ were extracted from the rate-level curves and plotted. $A$, Left hemisphere, contralateral to Cl. $\boldsymbol{B}$, Right hemisphere, ipsilateral to Cl. $\boldsymbol{C}$, Recording depths of Cl-driven and Cl-nonresponsive neurons, both hemispheres. Distributions of absolute recording depths (electrode tip at brain surface, either dura or tissue above dura, determined visually under microscope) and relative recording depths (from the first neuron encountered) are plotted along $y$-axis and $x$-axis, respectively, for each neuron. Because we approached the auditory cortex perpendicularly to the cortical surface, most well-isolated neurons encountered were recorded from more superficial layers. The median relative recording depths of $\mathrm{Cl}$-nonresponsive neurons was $0.4 \mathrm{~mm}$, whereas the median recording depth of $\mathrm{Cl}$ responsive neurons was significantly deeper, $0.56 \mathrm{~mm}$ (WRS test, $p=9.6 \mathrm{e}-7)$.

\section{CI stimulation selectively activates neural populations in A1}

Why are some neurons in auditory cortex nonresponsive to CI stimulation but others can be driven by it? Because most neurons analyzed in this report were responsive to acoustic stimulation, we were able to address this question by examining the acoustic response properties of neurons in either CI response category (referred to here as CI-driven and CI-nonresponsive, respectively). We hypothesized that there are some functional differences in a neuron's acoustic response properties that explain its CI responsiveness.

For most neurons, we measured frequency tuning at a single sound level, then measured a rate-level function at BF using acoustic stimuli. Figure $5 \mathrm{~A}$ shows representative examples of neurons with narrow or broad frequency tuning curves. The width of frequency tuning was quantified by a Q-value (see Materials and Methods), with lower Q-values reflecting broader tuning. Q-value distributions indicate that CI-driven neurons tended to have broader frequency tuning than CI-nonresponsive neurons (Fig. $5 B$, top; $p=2.74 \mathrm{e}-11$, WRS test). Cumulative distribution functions of the two populations show that CInonresponsive neurons had significantly larger Q-values and, therefore, sharper frequency tuning (Fig. 5B, bottom; $p=$ 3.52e-9, Kolmogorov-Smirnov test). Figure $5 C$ shows representative examples of neurons with nonmonotonic or monotonic rate-level functions. We used an MI to quantify the degree of monotonicity, which is defined as the ratio of firing rate at the loudest level tested to the maximum firing rate recorded at all levels (Sadagopan and Wang, 2008). Spontaneous firing rate was subtracted before the MI calculation. MI $=1$ indicates that a neuron had its highest firing at the loudest level tested, indicating a monotonic or saturating rate-level function. Low MI values approaching 0 indicate nonmonotonic tuning to sound level. Negative MI values reflect suppressed responses (below the spontaneous firing rate) at the highest sound level. CI-nonresponsive neurons tend to have lower MI values than CI-driven neurons, indicating they are more likely to have nonmonotonic rate-level 
functions than CI-driven neurons (Fig. $5 D$, top; $p=7.75 \mathrm{e}-9$, WRS test). Cumulative distribution functions (Fig. $5 D$, top) show that CI-nonresponsive neurons had significantly smaller MI values ( $p=$ 3.51e-11, Kolmogorov-Smirnov test). Neurons with nonmonotonic rate-level functions are more selective to sound level than neurons with monotonic rate-level functions (Sadagopan and Wang, 2008).

These results suggest that neurons that are more selective to frequency or sound level respond more weakly to CI stimulation. To further assess the relationship between acoustic receptive field characteristics of neurons and their CI responsiveness, we characterized a subset of neurons' FRAs by measuring frequency tuning curves across all sound levels. FRA is a commonly used measure of an auditory neuron's receptive field. Auditory nerve fibers have so-called "V-shaped" FRAs that become wider at higher sound levels (Kiang et al., 1965). While many studies in anesthetized animals have shown that neurons in A1 mostly have V-shaped FRAs (Clarey et al., 1992), experiments in awake animals have reported a large proportion of neurons in A1 to be narrowly tuned to both frequency and level, referred to as "O-shaped" FRAs (Sadagopan and Wang, 2008; Watkins and Barbour, 2011a).

An example of a CI-driven neuron is shown in Figure 6A. This neuron responded to tones at a wide range of frequencies as sound level increased, showing a "Vshaped" FRA (Fig. 6A, top right). Analogous to frequency and sound level, we tested the neuron's responses to electric stimuli at different electrodes and current levels and obtained a CI response area (Fig. 6A, bottom right), which showed a similar shape as the acoustic FRA. Figure $6 B$ shows FRAs of two other CI-driven neurons, both showing "V-shaped" FRAs. We found that CI stimulation was effective in activating predominantly those neurons with broad or V-shaped FRAs. In contrast, neurons nonresponsive to $\mathrm{CI}$ stimulation typically have "O-shaped" FRAs (Fig. 6C,D). These neurons respond to a narrow range of frequencies and show little or no response at the highest sound level tested. The neuron shown in Figure $6 \mathrm{C}$ did not respond to any electrode at any current levels tested (Fig. $6 C$, bottom left). Rather, this neuron was indeed suppressed by CI stimulation. Because the spontaneous firing of this neuron was low, it was not feasible to observe suppression with CI stimulation alone. However, when CI stimuli were presented at electrode 7-6 concurrently with the BF tone

\section{Frequency Tuning}

A

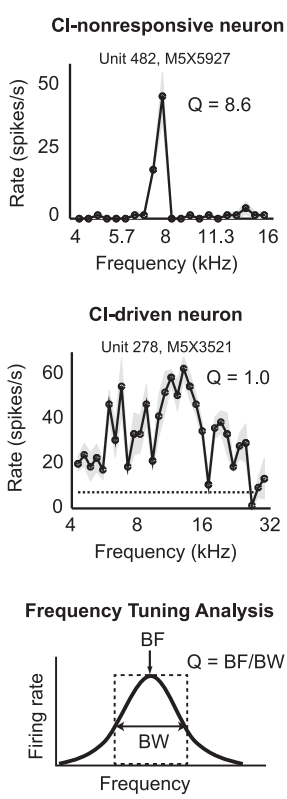

B
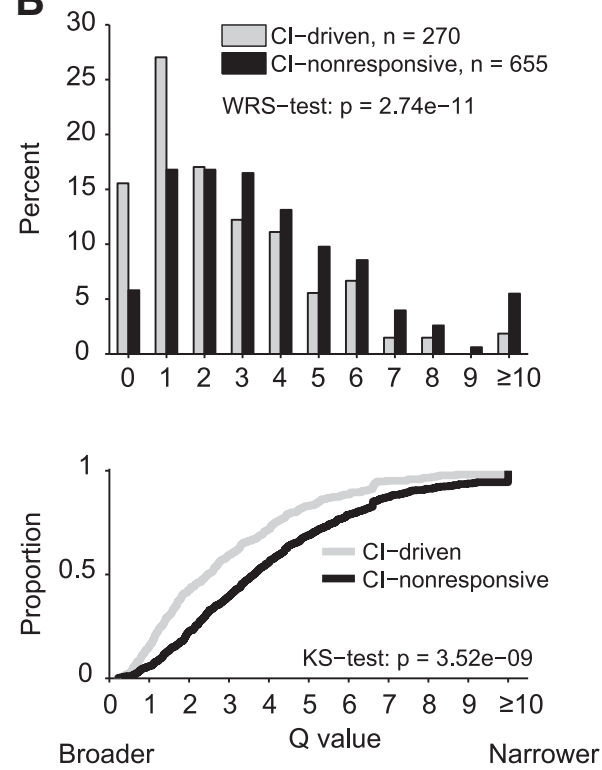

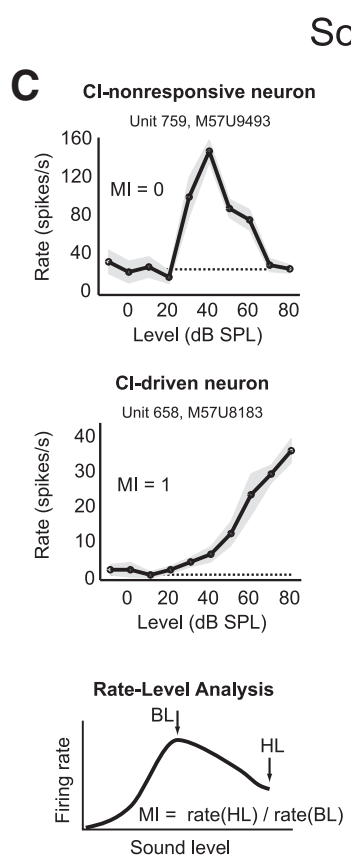

Sound Level Functions
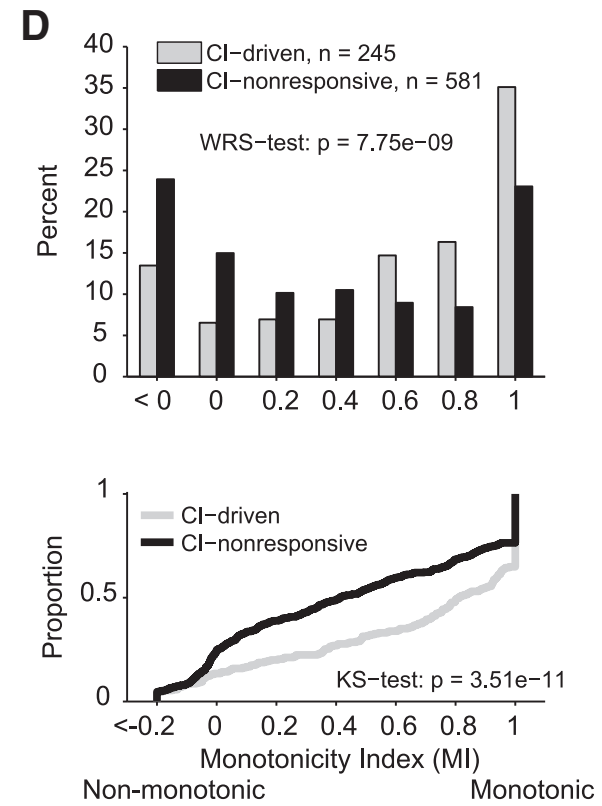

Figure 5. Cl-nonresponsive neurons exhibit narrower frequency tuning and greater nonmonotonicity over sound level. $A$, Exemplar frequency tuning curves from Cl-nonresponsive (top) and Cl-driven (middle) neurons, respectively. Dashed lines indicate spontaneous firing rate. Q-value calculation (bottom), calculated from acoustic frequency tuning curves. B, Top, Distributions of Q-values of Cl-driven and Cl-nonresponsive populations. Median Q-values are significantly different between the two populations (CI-driven: median Q-value $=2.49$, Cl-nonresponsive: median Q-value $=3.88, p=$ 2.74e-11, WRS test). Bottom, Cumulative distribution functions of the two populations show that (I-nonresponsive neurons had significantly larger Q-values, and therefore sharper frequency tuning ( $p=3.52 \mathrm{e}-9$, Kolmogorov-Smirnov test). Percentage of neurons from each animal: M57U (50\%), M77W (10\%), M5X (28\%), M3Y (12\%). C, Top, Middle, Examples of rate-level functions of $\mathrm{Cl}$-nonresponsive and $\mathrm{Cl}$-driven neurons, respectively. $\mathrm{MI}$ calculation (bottom), calculated from acoustic rate-level function. BL, Best level; $H L$, highest sound level tested. Spontaneous firing rate was subtracted from the mean firing rate before $\mathrm{MI}$ calculation. $\boldsymbol{D}$, Top, Distributions of $\mathrm{MI}$ for the two populations. Median MI was significantly different between the two populations ( $\mathrm{Cl}$-driven: median $\mathrm{MI}=0.81$, Cl-nonresponsive: median $\mathrm{MI}=0.42, p=7.75 \mathrm{e}-9$, WRS test). Bottom, Cumulative distribution functions show that Cl-nonresponsive neurons had significantly smaller MI values, and therefore greater nonmonotonicity in rate-level functions than $\mathrm{Cl}$-driven neurons ( $p=3.51 \mathrm{e}-11$, Kolmogorov-Smirnov test). Percentage of neurons from each animal: M57U (49\%), M77W (10\%), M5X (29\%), M3Y (12\%). 


\section{A}

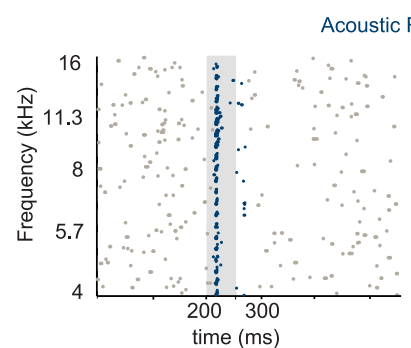

Cl Response (Electrode, Level)
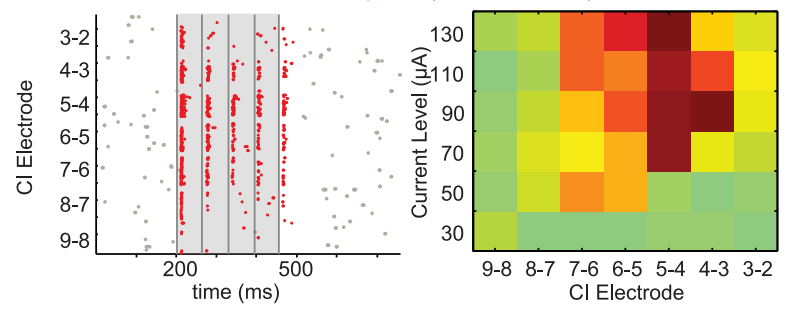

B

Examples of acoustic FRA (Cl-driven neurons, $\mathrm{SI}=1$ )
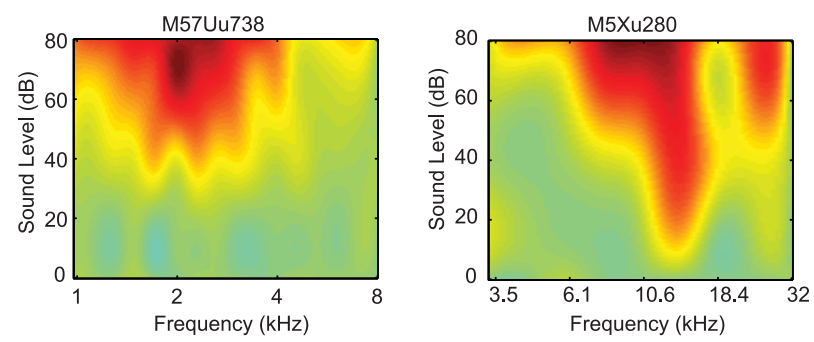

C

An example of a $\mathrm{Cl}$-nonresponsive neuron (M5Xu481)
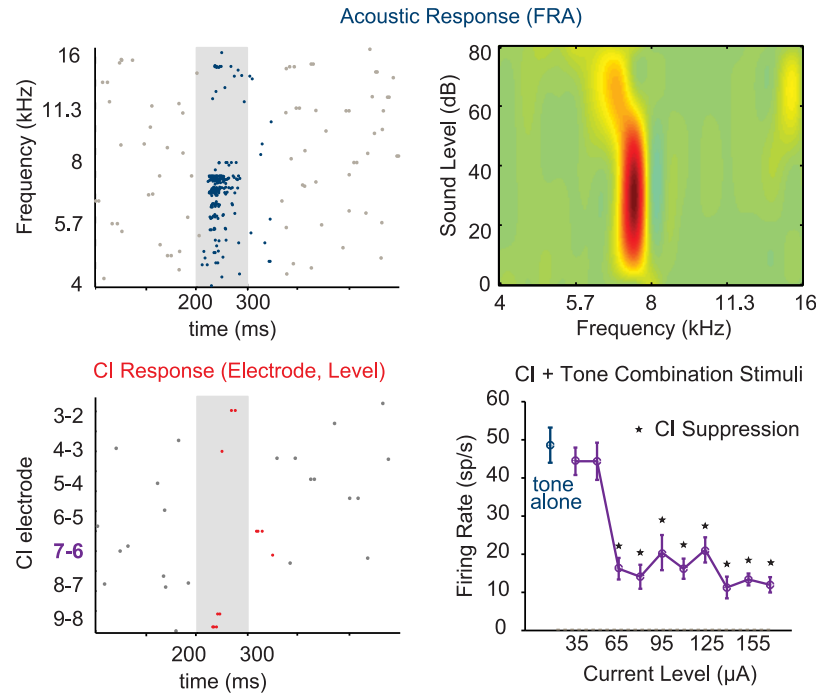

D

Examples of acoustic FRA ( $\mathrm{Cl}$-nonresponsive neurons, $\mathrm{SI}=0$ )
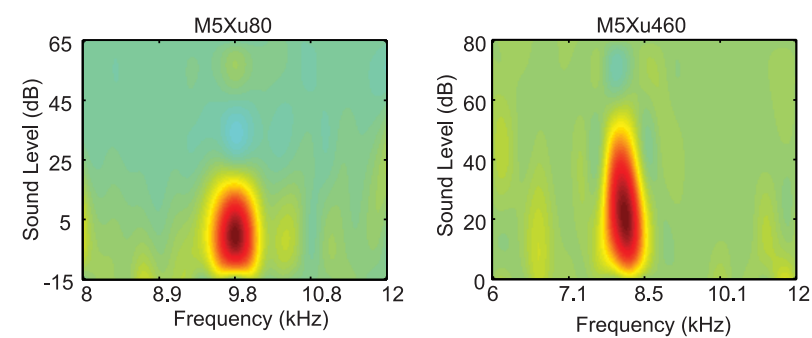

Figure 6. Frequency and Cl response areas of (I-driven and Cl-nonresponsive populations. A, Acoustic FRA in a Cl-driven neuron (top) and its Cl response area (bottom). This neuron has a "V-shaped" FRA, and responded to a broader range of frequencies as sound level increased. Shaded region represents the period of stimulation. Colored dots in the raster indicate spike times that fell within the analysis region for stimulus-driven response. The Cl response area shows this neuron's responses to $16 \mathrm{~Hz}$ electrical pulse trains delivered to different electrode channels and at different current levels. Dark lines in the raster plot indicate times of the $16 \mathrm{~Hz}$ pulse train stimulus. B, FRAs of two other (I-driven neurons. C, An example of a Cl-nonresponsive neuron's acoustic FRA (top). This neuron has an "0-shaped" FRA, with narrow frequency tuning and little or no response at the highest sound level tested. It did not respond to Cl stimuli (bottom, left, $257 \mathrm{~Hz} C \mathrm{Cl}$ stimulation). $\mathrm{Cl}+$ acoustic combination stimuli revealed significant Cl suppression (bottom, right). $\boldsymbol{D}$, Examples of two other (l-nonresponsive neurons.

that drove the neuron, firing rate decreased with increasing electric current level. This example indicates that such CI-nonresponsive neurons may actually be suppressed by CI stimuli.

We used an SI to quantify the FRA shape of all tested neurons (see Materials and Methods). An SI close to zero indicates an O-shaped FRA and a highly nonmonotonic rate-level function at $\mathrm{BF}$, whereas larger SI values indicate V-shaped FRAs (Kiang et al., 1965). The majority of CI-driven neurons had SI values close to 1 or greater, indicating V-shape FRAs (Fig. 7A). Conversely, the majority of CI-nonresponsive neurons have SI values close to zero, indicating O-shape FRAs (Fig. 7A). The median SI values of the two groups are significantly different (median SI: 1 for CI-driven, 0 for CI-nonresponsive, $p=1.67 \mathrm{e}-6$, WRS test). To further assess each neuron's selectivity to acoustic stimuli, another measure of FRA, response area (RA), was calculated. Briefly, each FRA is interpolated to have the same octave-step spacing, and the number of points with significant firing rate ( $>2$ SDs above mean spontaneous rate) is summed. CI-nonresponsive neurons have smaller RAs (median RA: 94.4 for CI-driven, 19.0 for CI-nonresponsive, $p=4.56 \mathrm{e}-8$, WRS test). SI and RA values were plotted for each neuron (Fig. $7 B$ ). CInonresponsive neurons are clustered in the lower left corner of the plot, further showing that CI stimulation is ineffective at activating A1 neurons that have smaller and more constrained FRA shapes.

\section{Acoustic sideband suppression predicts CI suppression}

There are several possible reasons that neurons with small and selective receptive fields are less likely to be activated by CI stimulation. First, the CI electrodes might not adequately stimulate the portion of the cochlea corresponding to a cortical neuron's $\mathrm{BF}$ due to the limited number of stimulation channels in the implanted CI electrode array. This is unlikely to be the primary explanation, however, because CI stimulation creates broad current spread in the cochlea, providing excitation to locations of the cochlea between electrodes (see also Fig. 9B,C). A more likely explanation relates to the finding that many $\mathrm{A} 1$ neurons are suppressed by off-BF frequency components (Ojima, 2011). This sideband suppression may result from intracortical inhibitory inputs or inhibition occurring at subcortical neurons that provide input to the A1 neuron. CI stimulation likely activates both excitatory and inhibitory frequency channels leading to a cortical neuron. Therefore, greater inhibitory drive relative to excitation may explain the lack of response to CI stimulation in an A1 neuron. This explanation is supported by the analyses illustrated in Figure 8, which shows that the majority of CI-nonresponsive neurons were actually suppressed by CI stimulation. Since the spontaneous firing of most A1 neurons is low in awake marmosets (median 2.57 spikes/s across sampled population; 29\% neu- 
A

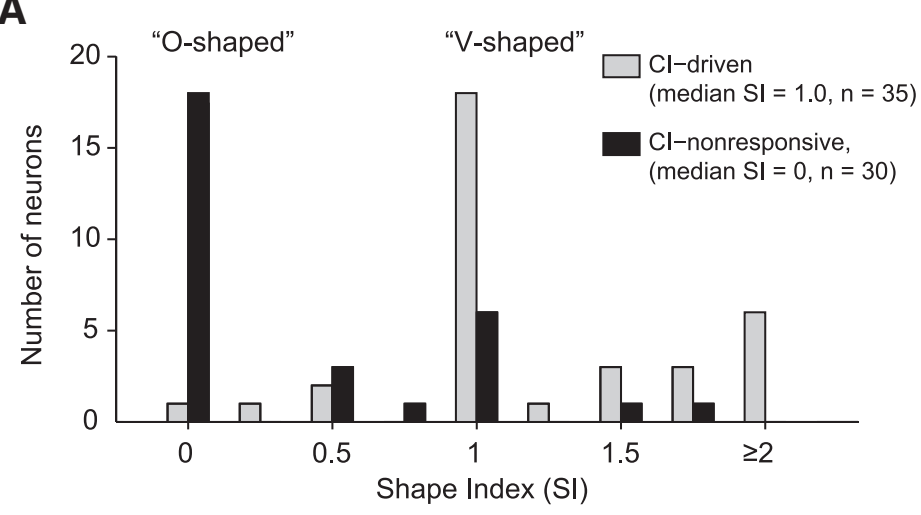

B

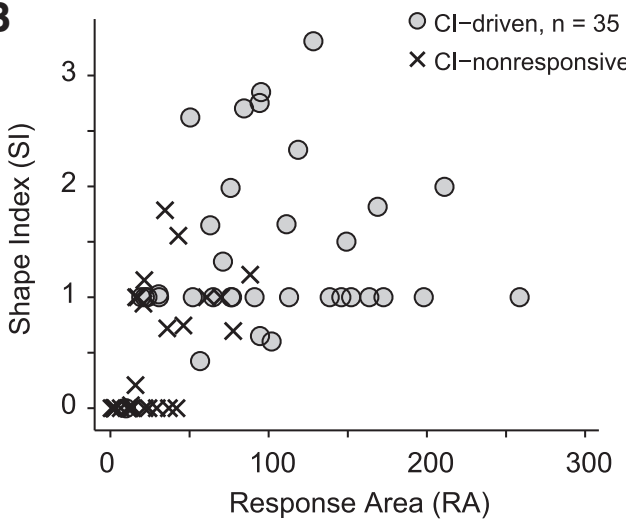

Figure 7. Quantification of frequency response areas. $A$, FRA shape is quantified by an SI. The two populations have significantly different $\mathrm{SI}$ values (CI-driven: median $\mathrm{SI}=1$, Cl-nonresponsive: median $\mathrm{SI}=0, p=1.67 \mathrm{e}-6$, WRS test). $\boldsymbol{B}$, (I-nonresponsive neurons have more constrained FRAs. RA, which quantifies the size of the FRA, is plotted against $\mathrm{SI}$. The majority of the 65 neurons shown in this figure came from the 2 animals that were neomycin-treated in the (I-implanted ear (M5X: 54\%; M3Y: 38\%). The remaining (8\%) was from another animal (M57U).

A

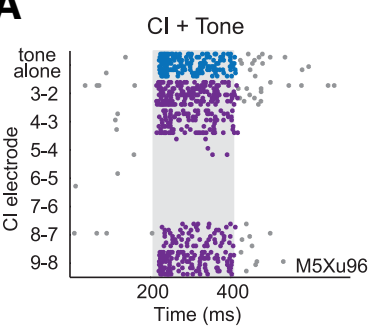

C
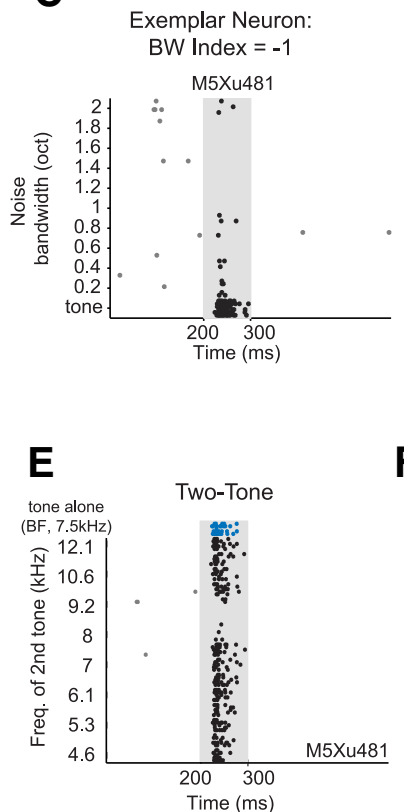

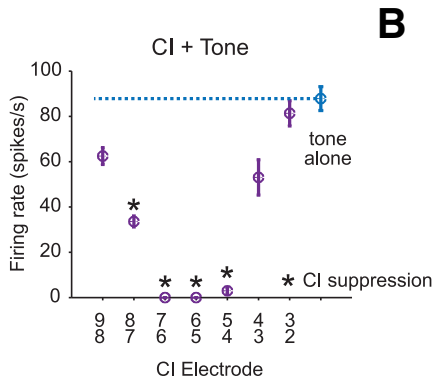

B

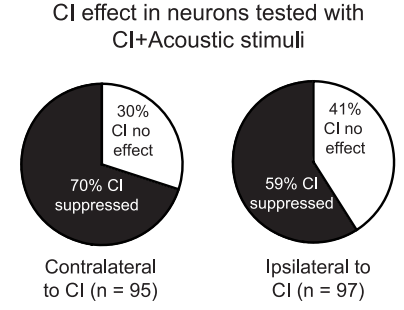

D
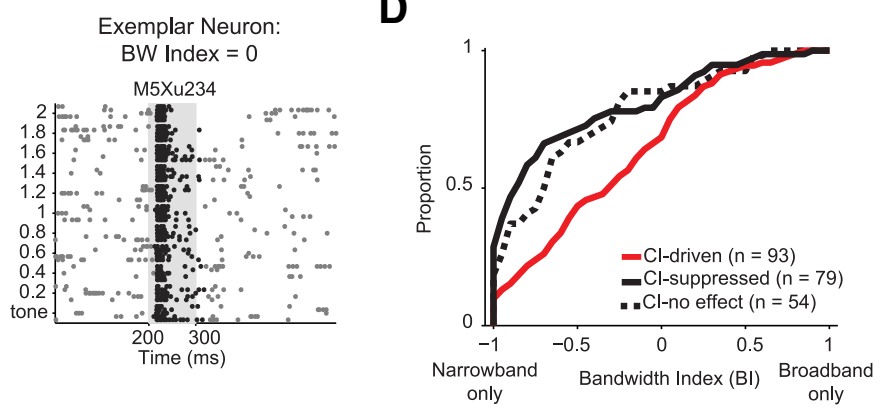

F

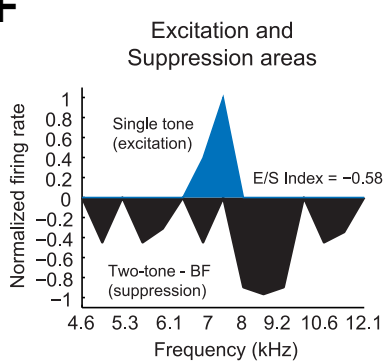

G

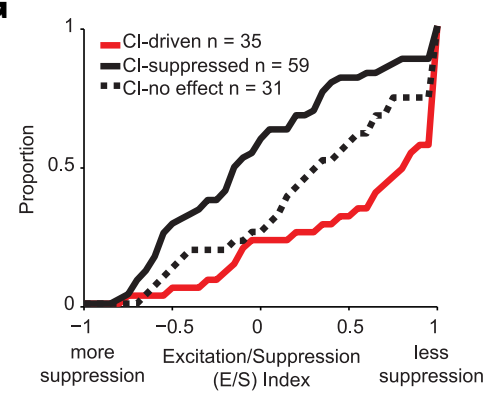

Figure 8. Cl suppression is associated with acoustic sideband suppression. $A$, Example of a neuron with Cl suppression, revealed by combined Cl + acoustic stimuli. Both dot raster (left) and firing rate profile (right) are shown. B, Proportions of Cl-nonresponsive neurons exhibiting (I suppression or no effect when tested by combined CI + acoustic stimuli. Percentage of neurons from each animal:M57U (7\%), M77W (16\%), M5X (57\%), M3Y (20\%). C, Examples of two neurons showing preference to narrowband stimuli (left) or no preference to bandwidth (right). Test stimuli were pure tone at BF and bandpass noises with varying bandwidth (centered at the BF). D, Cumulative distributions of the BI for (I-driven (median $=-0.26$ ) and (l-suppressed (median $=-0.84$ ). The medians of the two groups are significantly different $(p=8.24 \mathrm{e}-6$, WRS test). Percentage of neurons from each animal: M57U (11\%), M77W (22\%), M5X (55\%), M3Y (12\%). Percentage of neurons recorded from left and right hemispheres were 62\% and 48\%, respectively. E, Dot raster showing two-tone responses of a (l-suppressed neuron. The first tonewas at the neuron's BF ( $7.46 \mathrm{kHz}, 40 \mathrm{dBSPL}$ ). The frequency of the second tone ( $y$-axis) was varied from 4.6 to 12.1 $\mathrm{kHz}$ (40 dBSPL). For comparison, the response to BF-tonealone is also shown at the top of the raster plot (blue). $F$, Single-tone (blue) and two-tone (black, two-tone response - the BF response) frequency tuning curves, normalized to the BF response, were used to determine excitation and suppression areas, respectively, and calculate an E/S index (see Materials and Methods). Negative values indicate a large amount of suppression relative to the excitation area. G, Cumulative distributions of the E/S index for Cl-driven, (l-suppressed, and Cl-no effect populations of neurons. (I-driven neurons had significantly larger E/S index (median $=0.86$ ) than (l-suppressed neurons (median $=-0.09, p=1.18 \mathrm{e}-6$, WRS test). Percentage of neurons from each animal: M57U (21\%), M77W (8\%), M5X (43\%), M3Y (28\%). Percentage of neurons recorded from left and right hemispheres were $58 \%$ and $42 \%$, respectively. 
rons with spontaneous rate $<1$ spike/s), suppressive effects of CI stimulation alone can be difficult to observe. In a subset of CInonresponsive neurons, we presented a BF tone to the intact ear (to elicit firing) concurrently with a CI stimulus delivered to each electrode channel (Fig. 8A). As such, in these experiments, CI suppression is demonstrated by binaural suppression and serves as a proxy for sideband suppression seen in response to monaural stimulation. The neuron's response shown in Figure $8 A$ was significantly suppressed when the CI stimulation was applied to electrodes $8-7,7-6,6-5$, and 5-4. A significant drop of firing rate relative to the $\mathrm{BF}$ tone alone condition $(>50 \%$ rate reduction and $p<0.05$, WRS test) was considered CI suppression. Of 192 of CI-nonresponsive neurons tested in both hemispheres, CI stimulation suppressed $70 \%$ of 95 neurons tested in A1 contralateral to $\mathrm{CI}$, compared with $59 \%$ of 97 neurons tested in $\mathrm{A} 1$ ipsilateral to CI (Fig. 8B). These data indicate that CI stimulation suppresses similar proportions of neurons in the hemisphere ipsilateral or contralateral to CI, based on this combined acoustic + CI stimulation method of assessing suppression. This dataset also suggests that the large percentage of CI-nonresponsive neurons in the hemisphere ipsilateral to CI cannot be attributed to simple ipsilateral inhibition.

To further test the idea that broad current spread from CI electrical stimulation activates frequency channels suppressive to auditory cortex neurons, we systematically studied A1 neurons' responses to acoustic stimuli with varying bandwidths, ranging from pure tones to bandpass noises $(0-2$ octaves bandwidth, centered at BF). We assume that the broadband noise stimulus activates broad frequency place areas in the cochlea, more analogous (though obviously not identical) to CI stimulation. Two examples of neurons showing preferential responses to narrowband or broadband acoustic stimuli are illustrated in Figure $8 C$. To quantify a neuron's stimulus bandwidth preference, a BI was calculated (see Materials and Methods). Positive and negative values reflect preference for broadband and narrowband stimuli, respectively. A value near zero indicates that a neuron responds well to both broadband and narrowband stimuli. The CI-nonresponsive neurons were further separated into CIsuppressed and CI-no effect groups based on the test with CI and acoustic binaural combination stimuli described above (Fig. $8 A, B)$. We found that CI-driven neurons were more responsive to broadband acoustic stimuli than CI-suppressed neurons (median BI: -0.26 for CI-driven, -0.84 for CI-suppressed, $p=$ 8.24e-6, WRS test) or CI-no effect neurons (median BI: -0.65 for CI-no effect neurons, $p=0.001$, WRS test), although no significant difference was found between the CI-suppressed and CI-no effect distributions ( $p=0.428$, WRS test) (Fig. $8 D$ ).

The decrease in firing rate to broadband signals seen in many A1 neurons (e.g., neurons responsive to narrowband only, Fig. $8 C$, left) suggests that frequency components away from BF suppress their responses. To further examine possible mechanisms for the ineffective and suppressive effects of CI stimulation we observed, a subset of neurons were also tested with two-tone stimulation (Kadia and Wang, 2003). After the neuron's BF was determined from single-tone tuning, the BF tone was presented simultaneously with a second tone with frequency varied over a 1.5 octave range around the neuron's BF (Fig. $8 E$ ). Reductions in firing rate relative to the $\mathrm{BF}$-tone alone condition reveal suppression due to the second tone (Fig. $8 F$ ). The areas under each curve were used to calculate an E/S index (see Materials and Methods); a value of 1 indicates no significant two-tone suppression, whereas a negative value suggests a large amount of suppression relative to the excitation area. The ability of CI stimulation to activate a neuron is likely to depend on the balance of excitatory and inhibitory drive (Ojima, 2011). We therefore hypothesized that neurons with greater excitation areas and less two-tone suppression would be more likely to be driven by CI stimulation. The cumulative distributions of the E/S index were examined for CI-driven, CI-suppressed, and CI-no effect groups of neurons (Fig. $8 G$ ). We found that the group of CI-driven neurons had larger $\mathrm{E} / \mathrm{S}$ index (median 0.86 ) than the group of CI-suppressed neurons (median $=-0.09, p=1.18 \mathrm{e}-6$, WRS test). The distribution of CI-no effect neurons falls between the other two distributions (median 0.39, CI-no effect vs CI-suppressed, $p=$ 0.007; CI-no effect vs CI-driven, $p=0.059$, WRS test). These results, showing a greater extent of CI-induced suppression in neurons that are also suppressed by broadband acoustic stimuli and neurons with greater two-tone suppression (more negative $\mathrm{E} / \mathrm{S}$ index), provides a possible mechanistic explanation for the ineffectiveness of CI stimulation to activate certain A1 neurons.

\section{Effect of electrode configuration on neuronal responses}

In this study, we used a bipolar electrode configuration in which the active and return electrodes were adjacent contacts on the CI electrode array. A monopolar configuration consisting of an extracochlear return electrode is more commonly used clinically. In a substantial number of neurons we compared responses to monopolar (MP) and bipolar (BP) stimulation configurations. In these experiments, BP stimulation was typically the first modality tested, and neurons nonresponsive to BP were usually not further tested with MP. We found qualitatively similar responses to stimulation in the two modalities delivered at repetition rates $4-257 \mathrm{~Hz}$ (Fig. 9A). Differences in response strength and stimulus selectivity were observed when comparing CI response areas, derived from firing rate response to stimuli delivered to each electrode contact (MP) or contact pair (BP) across wide range of current levels (Fig. 9B). A larger number of stimulation channels in MP mode elicited responses compared with BP mode (Fig. 9C, median BP: 4 , median MP: 6, $p=2.63 \mathrm{e}-6$, WRS test), and maximum firing rate responses to MP were higher than BP (Fig. $9 D$, $p=3.37 \mathrm{e}-4$, right-tailed $t$ test). MP and BP CI response areas were similar in that the majority of neurons had "V-shape" response areas (Fig. $9 E$ ) and monotonic response to current level (Fig. $9 F$ ). We also assessed suppressive effects of BP and MP using combination $\mathrm{CI}+$ acoustic stimulation described earlier. We found that neurons suppressed by BP were almost always suppressed by MP, that the strength of suppression was greater, and that a greater number of stimulation contacts in MP mode suppressed responses compared with BP mode (median BP: $3, n=$ 115, median MP: $5, n=33, p=1.3 \mathrm{e}-4$, WRS test).

\section{Discussion}

\section{Neuronal selectivity and effectiveness of CI stimulation}

Previous neurophysiology studies in anesthetized animals have shown that CI stimulation causes broad activation of the auditory nerve (van den Honert and Stypulkowski, 1987; Kral et al., 1998), inferior colliculus (Snyder et al., 1990, 2004), and auditory cortex (Raggio and Schreiner, 1999; Bierer and Middlebrooks, 2002). The broad CI-evoked excitation reported by previous studies might lead to the expectation that the majority of A1 neurons in awake marmoset would respond to CI stimulation. However, we show in this study that CI stimulation is surprisingly ineffective at activating a large percentage of neurons in Al of awake marmoset. Moreover, the neurons that are activated by CI stimulation tend to be from different functional classes than those not acti- 
A

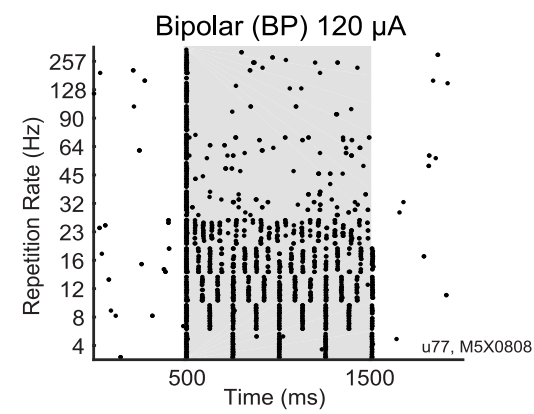

B

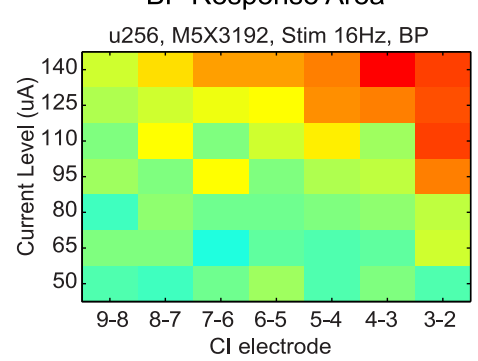

C

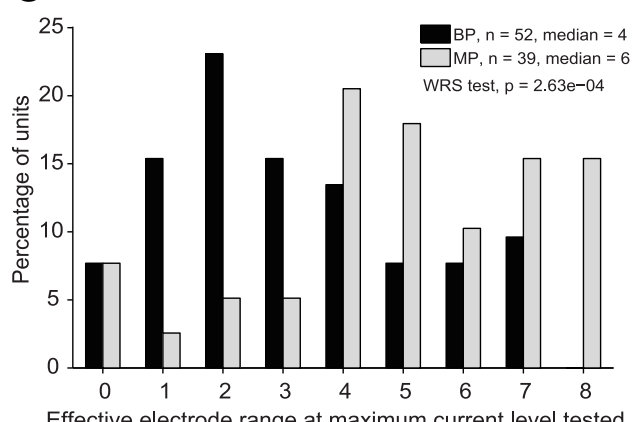

Effective electrode range at maximum current level tested

E

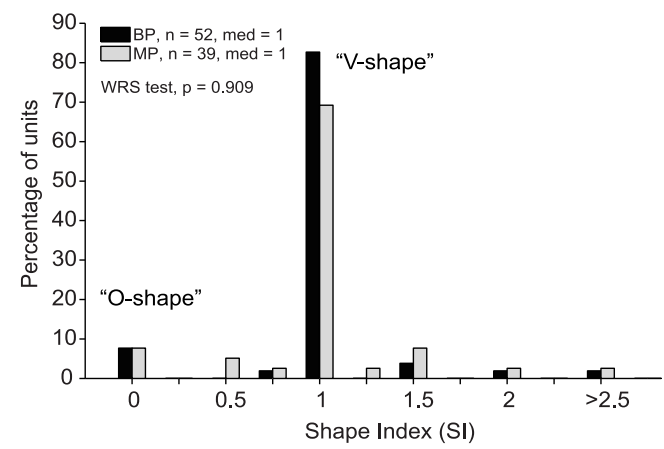

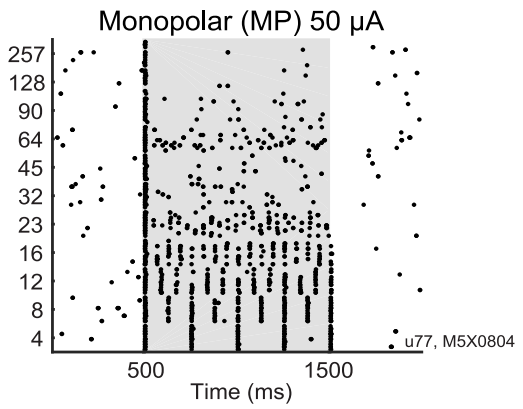

MP Response Area

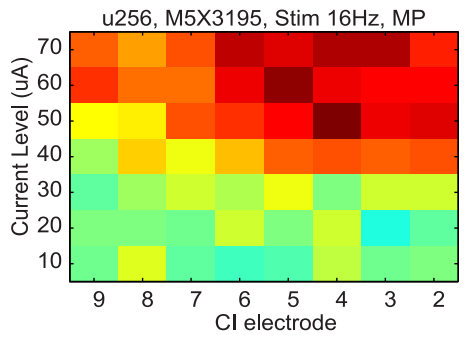

D

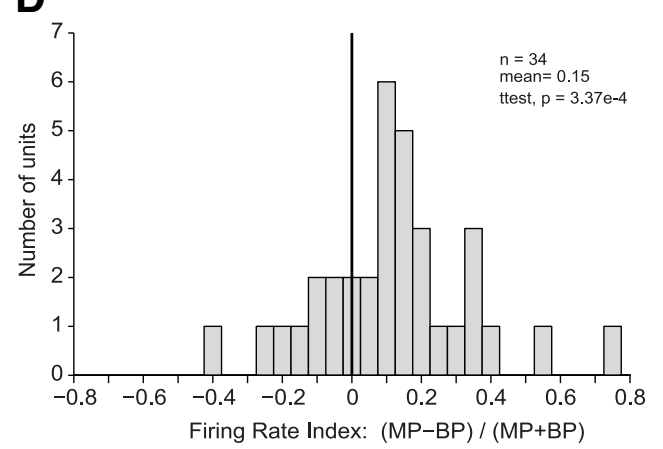

$\mathbf{F}$

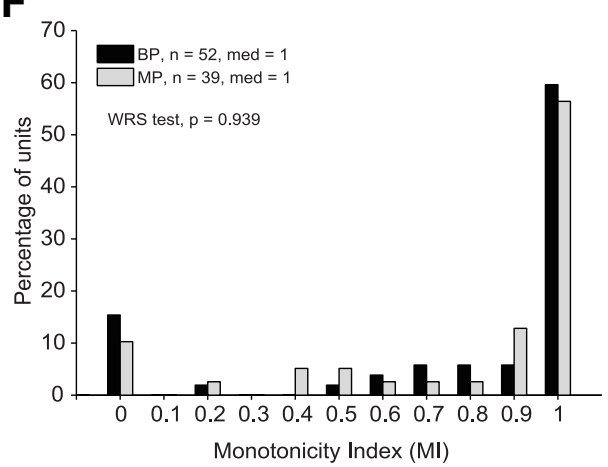

Figure 9. Effect of electrode configuration on neuronal responses. BP stimulation was between adjacent intracochlear electrode contacts. MP stimulation was between intracochlear contact and a distant electrode contact in the neck. Both stimulus configurations consisted of biphasic current pulses (100 $\mu$ s per phase, $50 \mu$ s interphase gap). MP stimulation required less current to reach neural thresholds. $A$, Representative example of neuron with similar response to BP and MP stimulation, with stimulus-synchronized response at slow repetition rates and transient onset response at high repetition rates. $\boldsymbol{B}$, Response areas of an example neuron; BP response area on the left, MP response areas on the right. Analogous to acoustic FRAs, Cl response areas reflect the firing rate response to $\mathrm{Cl}$ stimuli delivered to each electrode in the array at a wide range of current levels. Heat maps reflect the firing rate at each electrode-current combination. $C$, Distributions of effective electrode range for $\mathrm{BP}$ and $\mathrm{MP}$ configurations. Effective electrode range was defined as the number of electrodes with a stimulus-driven response at the maximum current level tested. The two distributions were significantly different, with MP causing larger effective electrode range than $\mathrm{BP}(p=2.83 \mathrm{e}-4$, WRS test, median $\mathrm{BP}=4$, median $\mathrm{MP}=6)$. $D$, Distribution of firing rate index in neurons tested with both $B P$ and MP. Maximum firing rates in the $B P$ and MP response areas, respectively, were used to calculate the firing rate index. A positive value indicates higher firing rate evoked by MP compared with BP. The mean firing rate index was 0.15 , and the distribution was significantly $>0(p=3.37 \mathrm{e}-4$, right tailed $t$ test $)$. $E$, Distributions of $S I$ of $B P$ and MP response areas. $S I$ was calculated to be ratio of effective electrode range at the highest current level to the effective electrode range at the best current level. The median SI value for both BP and MP response areas was 1, reflecting "V-shape" response areas, and there was no difference between the two distributions ( $p=0.909$, WRS test). $\boldsymbol{F}$, Distributions of Ml for BP and MP rate-level functions taken from the response areas. Ml was calculated as the ratio of the firing rate at maximum current level to the firing rate at the best current level. MI values $>0.5$ are considered to be monotonic. A value of 1 indicates that the maximum current level tested evoked maximum firing. The median MI value for both BP and MP was 1 , and there was no difference between the two distributions ( $p=0.939$, WRS test). 
vated by CI stimulation, as defined by their acoustic response properties. Neurons with nonmonotonic response ("O-shaped" neurons if classified by FRA) to sound level make up a large proportion of neurons in awake monkey auditory cortex (Sadagopan and Wang, 2008; Watkins and Barbour, 2011a), and these neurons are consistently not activated by CI stimulation. What are the implications of weak activation of such selective neural populations by CI stimulation? Two aspects of auditory perception that might be adversely affected are dynamic range and hearing in noise. Compared with normal hearing listeners, CI users experience a more limited dynamic hearing range (Zeng et al., 2002; Moore, 2003), can discriminate fewer steps in sound amplitude across the dynamic range (Schroder et al., 1994), and have greater difficulty understanding speech in noisy environments (Friesen et al., 2001; Stickney et al., 2004). In normal hearing, the perception of sound objects can remain relatively constant over a large range of sound levels, and it has been suggested that O-shaped neurons could contribute to such robust representations (Sadagopan and Wang, 2008). Although individual O-shaped neurons may have limited dynamic ranges, Sadagopan and Wang (2008) found that as a population their response areas span almost uniformly across sound level. O-shaped neurons may also be particularly well suited for encoding sound in noise. Neurons with nonmonotonic tuning have been shown to retain sensitivity to soft sounds in stimuli with high overall sound level (Watkins and Barbour, 2008, 2011b), and contribute to robust auditory object recognition in noisy conditions (Barbour, 2011).

The differences in CI implant procedures ( 2 of 4 animals ototoxically deafened) may create a potential confound in this study. Several studies have shown that neuronal responses in the auditory nerve (Sato et al., 2016) and inferior colliculus (Miller et al., 2006) to electrical stimulation can differ in animals with intact hair cells. We do not discount the possibility that some aspects of neuronal responses would be different across groups of animals given the possibility of electrophonic stimulation of residual hair cells not destroyed during electrode insertion in nondeafened animals. However, the percentages of CI-nonresponsive units (Fig. 3A), as well as the statistical tests performed between groups of primary measures (Q-value, MI, SI, BW index, E/S ratio) suggest that the patterns of A1 responsiveness were similar between the two groups of animals.

\section{Asymmetric activation of auditory cortex by unilateral CI stimulation}

One of the most striking observations we made in this study was the inability of electrical stimulation to drive auditory neurons in the hemisphere ipsilateral to the CI device. In all 4 animals, $<12 \%$ of neurons in ipsilateral A1 responded to CI stimuli, compared with $20 \%-45 \%$ in the contralateral hemisphere. This is in sharp contrast to the bilateral cortical activation we observed in animals receiving primarily monaural acoustic input. What are possible explanations for asymmetric activation by CI stimulation? Anatomical studies suggest that contralateral auditory projections are more numerous and stronger than ipsilateral projections (Brunso-Bechtold et al., 1981; Nordeen et al., 1983). Unless plastic reorganization occurred shortly after CI implantation in the animals observed in this study, this explanation based on anatomical connections is unlikely given our findings of bilateral activation from monaural acoustic stimulation. Because binaural integration begins early in the auditory pathway, starting in the cochlear nucleus and continuing at each subsequent processing stage
(Grothe et al., 2010), it is not surprising that many neurons in both left and right auditory cortex respond to monaural acoustic stimulation. Several physiological studies have found monaural acoustic stimulation to be effective in activating ipsilateral auditory cortex neurons (Brugge and Merzenich, 1973; Cheung et al., 2009); however, no studies in nonhuman primates have specifically examined the proportion of A1 neurons responsive to monaural acoustic stimulation of each of the two ears.

Another explanation for the asymmetric activation by CI stimulation could be that the CI stimulation drives inhibitory pathways more effectively than acoustic stimuli. The preponderance of CIsuppressed responses we found (64\% of CI-nonresponsive neurons tested) supports inhibition as a mechanism to explain the paucity of CI-driven responses. Yet we found no evidence that CI suppression was more prevalent in the hemisphere ipsilateral to the CI. Indeed, the opposite was true: CI stimulation suppressed $70 \%$ of neurons in left (contralateral) A1, compared with 59\% in right (ipsilateral) A1 (Fig. $8 B$ ). This result does not rule out stronger ipsilateral inhibition, however. The site of inhibition may occur elsewhere in the auditory pathway, such as subcortical nuclei or in other neurons in the cortical network that input to the neurons we recorded (the majority of neurons we record are likely from cortical layers $2 / 3$; see Materials and Methods). For example, the IC has prominent projections to ipsilateral thalamus and subsequently cortex, and can be strongly inhibited by stimulation of the ipsilateral ear (Pollak et al., 2002).

Recent human (Gordon et al., 2013) and animal (Kral et al., 2013a, b) studies demonstrate that extended monaural hearing leads to functional reorganization of auditory cortex and preference for the hearing ear. Kral et al. (2013a,b) showed that electrical stimulation of the hearing ear caused strong bilateral activation (as measured by surface local field potentials, whereas stimulation of the deaf ear resulted in overall weak cortical activation and contralateral dominance. The results from this study, with stimulation of the CI-implanted ear causing asymmetric (and overall weak) cortical activation and acoustic stimulation of the intact hearing ear causing strong bilateral activation, are loosely analogous to the Kral et al. (2013a,b) studies.

Studies in CI patients have demonstrated varying degrees of asymmetric cortical activation from monaural CI stimulation. Several PET studies in CI users have shown dominant contralateral activation (Naito et al., 1995; Hirano et al., 1997), although one PET study in CI subjects early after implantation ( $<2$ months) showed ipsilateral dominance of CI stimulation (Ito et al., 2004). There is evidence that in some users unilateral CI stimulation causes bilateral activation in auditory cortex (Herzog et al., 1991; Limb et al., 2010). A number of studies using PET (Fujiki et al., 1999; Giraud et al., 2001; Green et al., 2005; Mortensen et al., 2006) and auditory evoked potentials (Zhang et al., 2010; Sharma et al., 2011) suggest that the extent and strength of cortical activation are associated with CI performance. Naito et al. (1997) and Green et al. (2005) have shown CI users with poor speech recognition through their device have less activation in auditory association areas than control subjects and CI users with good speech (Naito et al., 1997; Green et al., 2005). Green et al. (2005, 2008) showed bilateral cortical activation of good performers, whereas poor performers showed much less (mostly contralateral) activation (Green et al., 2005, 2008). Nourski et al. (2013) recorded intracranially recorded auditory cortical responses to CI stimulation in a neurosurgical patient and reported that changes in location of intracochlear stimulation were perceived by the subject but not paralleled by changes in the 
spatial distribution of cortical activity. In contrast, in normalhearing individuals, spatial activation patterns elicited by pure tone stimuli typically vary as a function of stimulus frequency. In this context, we might consider the animal preparation used in this study analogous to a "poor" CI user. We hypothesize that, if an animal received chronic behaviorally relevant CI stimulation, the proportion of neurons responsive to CI stimulation would increase and result in more bilateral activation.

The results presented in this study come with an important caveat: the animals used in this study received CI stimulation only during experimental sessions. Further studies are needed to test whether animals receiving chronic, behaviorally relevant CI stimulation would show similar lack of response of $\mathrm{O}$-shaped neurons in $\mathrm{A} 1$. One may speculate that $\mathrm{CI}$ users who are better at certain psychophysical tasks, such as speech recognition in noise, are better able to recruit these selective neuronal populations (i.e., O-shaped neurons). This could take place due to plasticity driven by experience and learning with the CI device (Wilson et al., 2011; Kral and Sharma, 2012). These neurons are an attractive target for investigation of how neuronal responses change in A1 as an implant user gains experience with using CI input for goal-oriented hearing, such as communication. We believe that, to truly understand the neural underpinnings of CIs, one must know how the CI stimulation engages (or fails to engage) the auditory cortex before the plasticity takes place. The novelty and contribution of the present work are that it points out where information could be missing in auditory cortex with singlechannel CI stimulation. Our study is the first that links the effectiveness of CI stimulation to neuronal selectivity in auditory cortex. No previous studies have revealed such detailed information at the level of single neurons in the auditory cortex of awake primates.

\section{Implications for cortical processing of unilateral CI stimulation in single-sided deafness population}

The unilateral deafness model created in the marmoset in this study (in 2 of 4 animals) has important clinical relevance. In recent years, there has been a rapidly increased interest in treating patients with single-sided deafness with a unilateral CI implant on the deaf ear (Tokita et al., 2014). Single-sided deafness occurs in $\sim 0.5$ per 1000 newborns (Watkin and Baldwin, 2012; Kral et al., 2013a), 2-5 per 1000 children (Hassepass et al., 2013), and $\sim 1$ per 10,000 adults (Vincent et al., $2015)$. In the United States, $\sim 60,000$ people acquire singlesided deafness every year (Weaver, 2015). Our unilaterally deafened marmosets provide a valuable model to study how acoustically and electrically conveyed information interacts in auditory cortex when a subject with single-sided deafness is implanted with a CI device. Because most of A1 neurons are binaural (Middlebrooks et al., 1980), it is important to understand how cortical neurons integrate information conveyed acoustically and electrically via the two ears. Combined acoustic and CI stimulation can result in either suppressive (Fig. 8A) or facilitatory (data not shown) responses in A1 neurons. The interaction between acoustic and CI stimulation appears to be asymmetric (i.e., CI stimulation was primarily inhibitory on acoustically evoked responses) and nonlinear (i.e., the response evoked by combined stimulation was not a linear superposition of monaurally evoked responses).

\section{References}

Barbour DL (2011) Intensity-invariant coding in the auditory system. Neurosci Biobehav Rev 35:2064-2072. CrossRef Medline
Bendor D, Wang X (2005) The neuronal representation of pitch in primate auditory cortex. Nature 436:1161-1165. CrossRef Medline

Bierer JA, Middlebrooks JC (2002) Auditory cortical images of cochlearimplant stimuli: dependence on electrode configuration. J Neurophysiol 87:478-492. Medline

Blamey P, Artieres F, Başkent D, Bergeron F, Beynon A, Burke E, Dillier N, Dowell R, Fraysse B, Gallégo S, Govaerts PJ, Green K, Huber AM, KleinePunte A, Maat B, Marx M, Mawman D, Mosnier I, O'Connor AF, O'Leary S, et al. (2013) Factors affecting auditory performance of postlinguistically deaf adults using cochlear implants: an update with 2251 patients. Audiol Neurotol 18:36-47. CrossRef

Brugge JF, Merzenich MM (1973) Responses of neurons in auditory cortex of the macaque monkey to monaural and binaural stimulation. J Neurophysiol 36:1138-1158. Medline

Brunso-Bechtold JK, Thompson GC, Masterton RB (1981) HRP study of the organization of auditory afferents ascending to central nucleus of inferior colliculus in cat. J Comp Neurol 197:705-722. CrossRef Medline

Cheung SW, Bonham BH, Schreiner CE, Godey B, Copenhaver DA (2009) Realignment of interaural cortical maps in asymmetric hearing loss. J Neurosci 29:7065-7078. CrossRef Medline

Chung Y, Hancock KE, Nam SI, Delgutte B (2014) Coding of electric pulse trains presented through cochlear implants in the auditory midbrain of awake rabbit: comparison with anesthetized preparations. J Neurosci 34: 218-231. CrossRef Medline

Clarey JC, Barone P, Imig TJ (1992) Physiology of thalamus and cortex. In: The mammalian auditory pathway: neurophysiology (Popper AN, Fay RR, eds), pp 232-334. New York: Springer.

de la Mothe LA, Blumell S, Kajikawa Y, Hackett TA (2006) Cortical connections of the auditory cortex in marmoset monkeys: core and medial belt regions. J Comp Neurol 496:27-71. CrossRef Medline

de Villers-Sidani E, Merzenich MM (2011) Lifelong plasticity in the rat auditory cortex: basic mechanisms and role of sensory experience. Prog Brain Res 191:119-131. CrossRef Medline

Fallon JB, Shepherd RK, Irvine DR (2014) Effects of chronic cochlear electrical stimulation after an extended period of profound deafness on primary auditory cortex organization in cats. Eur J Neurosci 39:811-820. CrossRef Medline

Friesen LM, Shannon RV, Baskent D, Wang X (2001) Speech recognition in noise as a function of the number of spectral channels: comparison of acoustic hearing and cochlear implants. J Acoust Soc Am 110:1150-1163. CrossRef Medline

Fujiki N, Naito Y, Hirano S, Kojima H, Shiomi Y, Nishizawa S, Konishi J, Honjo I (1999) Correlation between rCBF and speech perception in cochlear implant users. Auris Nasus Larynx 26:229-236. CrossRef Medline

Giraud AL, Price CJ, Graham JM, Truy E, Frackowiak RS (2001) Crossmodal plasticity underpins language recovery after cochlear implantation. Neuron 30:657-663. CrossRef Medline

Gordon KA, Wong DD, Papsin BC (2013) Bilateral input protects the cortex from unilaterally-driven reorganization in children who are deaf. Brain 136:1609-1625. CrossRef Medline

Green KM, Julyan PJ, Hastings DL, Ramsden RT (2005) Auditory cortical activation and speech perception in cochlear implant users: effects of implant experience and duration of deafness. Hear Res 205:184-192. CrossRef Medline

Green KM, Julyan PJ, Hastings DL, Ramsden RT (2008) Auditory cortical activation and speech perception in cochlear implant users. J Laryngol Otol 122:238-245. Medline

Grothe B, Pecka M, McAlpine D (2010) Mechanisms of sound localization in mammals. Physiol Rev 90:983-1012. CrossRef Medline

Hassepass F, Aschendorff A, Wesarg T, Kröger S, Laszig R, Beck RL, Schild C, Arndt S (2013) Unilateral deafness in children: audiologic and subjective assessment of hearing ability after cochlear implantation. Otol Neurotol 34:53-60. CrossRef Medline

Herzog H, Lamprecht A, Kühn A, Roden W, Vosteen KH, Feinendegen LE (1991) Cortical activation in profoundly deaf patients during cochlear implant stimulation demonstrated by H2(15)O PET. J Comput Assist Tomogr 15:369-375. CrossRef Medline

Hirano S, Naito Y, Okazawa H, Kojima H, Honjo I, Ishizu K, Yenokura Y, Nagahama Y, Fukuyama H, Konishi J (1997) Cortical activation by monaural speech sound stimulation demonstrated by positron emission tomography. Exp Brain Res113:75-80. Medline

Ito K, Momose T, Oku S, Ishimoto S, Yamasoba T, Sugasawa M, Kaga K 
(2004) Cortical activation shortly after cochlear implantation. Audiol Neurootol 9:282-293. CrossRef Medline

Johnson LA, Della Santina CC, Wang X (2012) Temporal bone characterization and cochlear implant feasibility in the common marmoset (Callithrix jacchus). Hear Res 290:37-44. CrossRef Medline

Kadia SC, Wang X (2003) Spectral integration in Al of awake primates: neurons with single- and multipeaked tuning characteristics. J Neurophysiol 89:1603-1622. CrossRef Medline

Kennedy DW (1987) Multichannel intracochlear electrodes: mechanism of insertion trauma. Laryngoscope 97:42-49. Medline

Kiang NY, Watanabe EC, Clark LF (1965) Discharge patterns of single fibers in the cat's auditory nerve. Cambridge, MA: Massachusetts Institute of Technology.

Kim D, Gopalakrishna V, Guo S, Lee H, Torlak M, Kehtarnavaz N, Lobo A, Loizou PC (2009) On the design of a flexible stimulator for animal studies in auditory prostheses. Biomed Signal Process Control 2009:1-5. CrossRef Medline

Kirby A, Middlebrooks J (2012) Unanesthetized auditory cortex exhibits multiple codes for gaps in cochlear implant pulse trains. J Assoc Resin Otolaryngol 13:67-80. CrossRef Medline

Kral A, Sharma A (2012) Developmental neuroplasticity after cochlear implantation. Trends Neurosci 35:111-122. CrossRef Medline

Kral A, Hartmann R, Mortazavi D, Klinke R (1998) Spatial resolution of cochlear implants: the electrical field and excitation of auditory afferents. Hear Res 121:11-28. CrossRef Medline

Kral A, Hubka P, Heid S, Tillein J (2013a) Single-sided deafness leads to unilateral aural preference within an early sensitive period. Brain 136: 180-193. CrossRef Medline

Kral A, Heid S, Hubka P, Tillein J (2013b) Unilateral hearing during development: hemispheric specificity in plastic reorganizations. Front Syst Neurosci 7:93. CrossRef Medline

Limb CJ, Molloy AT, Jiradejvong P, Braun AR (2010) Auditory cortical activity during cochlear implant-mediated perception of spoken language, melody, and rhythm. J Assoc Resin Otolaryngol 11:133-143. CrossRef Medline

Lu T, Liang L, Wang X (2001) Neural representations of temporally asymmetric stimuli in the auditory cortex of awake primates. J Neurophysiol 85:2364-2380. Medline

McDermott HJ (2004) Music perception with cochlear implants: a review. Trends Amplif 8:49-82. CrossRef Medline

Middlebrooks JC, Dykes RW, Merzenich MM (1980) Binaural responsespecific bands in primary auditory cortex (AI) of the cat: topographical organization orthogonal to isofrequency contours. Brain Res181:31-48. CrossRef Medline

Middlebrooks JC, Bierer JA, Snyder RL (2005) Cochlear implants: the view from the brain. Curr Opin Neurobiol 15:488-493. CrossRef Medline

Miller CA, Abbas PJ, Robinson BK, Nourski KV, Zhang F, Jeng FC (2006) Electrical excitation of the acoustically sensitive auditory nerve: singlefiber responses to electric pulse trains. J Assoc Res Otolaryngol 7:195-210. CrossRef Medline

Moore BC (2003) Coding of sounds in the auditory system and its relevance to signal processing and coding in cochlear implants. Otol Neurotol 24: 243-254. CrossRef Medline

Moore BC (2012) An introduction to the psychology of hearing. Bingley, United Kingdom: Emerald.

Moore DR, Shannon RV (2009) Beyond cochlear implants: awakening the deafened brain. Nat Neurosci 12:686-691. CrossRef Medline

Mortensen MV, Mirz F, Gjedde A (2006) Restored speech comprehension linked to activity in left inferior prefrontal and right temporal cortices in postlingual deafness. Neuroimage 31:842-852. CrossRef Medline

Naito Y, Okazawa H, Honjo I, Hirano S, Takahashi H, Shiomi Y, Hoji W, Kawano M, Ishizu K, Yonekura Y (1995) Cortical activation with sound stimulation in cochlear implant users demonstrated by positron emission tomography. Cogn Brain Res 2:207-214. CrossRef Medline

Naito Y, Hirano S, Honjo I, Okazawa H, Ishizu K, Takahashi H, Fujiki N, Shiomi Y, Yonekura Y, Konishi J (1997) Sound-induced activation of auditory cortices in cochlear implant users with post- and prelingual deafness demonstrated by positron emission tomography. Acta Otolaryngol 117:490-496. CrossRef Medline

Ni D, Shepherd RK, Seldon HL, Xu SA, Clark GM, Millard RE (1992) Cochlear pathology following chronic electrical stimulation of the auditory nerve: I. Normal hearing kittens. Hear Res 62:63-81. CrossRef Medline
Nordeen KW, Killackey HP, Kitzes LM (1983) Ascending auditory projections to the inferior colliculus in the adult gerbil, Meriones unguiculatus. J Comp Neurol 214:131-143. CrossRef Medline

Nourski KV, Etler CP, Brugge JF, Oya H, Kawasaki H, Reale RA, Abbas PJ, Brown CJ, Howard MA 3rd (2013) Direct recordings from the auditory cortex in a cochlear implant user. J Assoc Res Otolaryngol 14:435-450. CrossRef Medline

Nuttall AL, Marques DM, Lawrence M (1977) Effects of perilymphatic perfusion with neomycin on the cochlear microphonic potential in the guinea pig. Acta Otolaryngol 83:393-400. CrossRef Medline

Ojima H (2011) Interplay of excitation and inhibition elicited by tonal stimulation in pyramidal neurons of primary auditory cortex. Neurosci Biobehav Rev 35:2084-2093. CrossRef Medline

Osmanski MS, Wang X (2011) Measurement of absolute auditory thresholds in the common marmoset (Callithrix jacchus). Hear Res 277:127133. CrossRef Medline

Pistorio AL, Vintch B, Wang X (2006) Acoustic analysis of vocal development in a New World primate, the common marmoset (Callithrix jacchus). J Acoust Soc Am 120:1655-1670. CrossRef Medline

Pollak GD, Burger RM, Park TJ, Klug A, Bauer EE (2002) Roles of inhibition for transforming binaural properties in the brainstem auditory system. Hear Res 168:60-78. CrossRef Medline

Pyman B, Blamey P, Lacy P, Clark G, Dowell R (2000) The development of speech perception in children using cochlear implants: effects of etiologic factors and delayed milestones. Am J Otol 2000 21:57-61. Medline

Raggio MW, Schreiner CE (1999) Neuronal responses in cat primary auditory cortex to electrical cochlear stimulation: III. Activation patterns in short- and long-term deafness. J Neurophysiol 82:3506-3526. Medline

Sadagopan S, Wang X (2008) Level invariant representation of sounds by populations of neurons in primary auditory cortex. J Neurosci 28:34153426. CrossRef Medline

Sadagopan S, Wang X (2009) Nonlinear spectrotemporal interactions underlying selectivity for complex sounds in auditory cortex. J Neurosci 29:11192-11202. CrossRef Medline

Sato M, Baumhoff P, Kral A (2016) Cochlear implant stimulation of a hearing ear generates separate electrophonic and electroneural responses. J Neurosci 36:54-64. CrossRef Medline

Schroder AC, Viemeister NF, Nelson DA (1994) Intensity discrimination in normal-hearing and hearing-impaired listeners. J Acoust Soc Am 96: 2683-2693. CrossRef Medline

Shannon RV (2012) Advances in auditory prostheses. Curr Opin Neurol 25:61-66. CrossRef Medline

Sharma A, Cardon G, Henion K, Roland P (2011) Cortical maturation and behavioral outcomes in children with auditory neuropathy spectrum disorder. Int J Audiol 50:98-106. CrossRef Medline

Skinner MW, Clark GM, Whitford LA, Seligman PM, Staller SJ, Shipp DB, Shallop JK, Everingham C, Menapace CM, Arndt PL, Antogenelli T, Brimacombe JA, Pijl S, Daniels P, George CR, Mcdermott HJ, Beiter AL (1994) Evaluation of a new spectral peak coding strategy for the nucleus 22 channel cochlear implant system. Am J Otol 15:15-27. Medline

Snyder RL, Rebscher SJ, Cao KL, Leake PA, Kelly K (1990) Chronic intracochlear electrical stimulation in the neonatally deafened cat: I. Expansion of central representation. Hear Res 50:7-33. CrossRef Medline

Snyder RL, Bierer JA, Middlebrooks JC (2004) Topographic spread of inferior colliculus activation in response to acoustic and intracochlear electric stimulation. J Assoc Resin Otolaryngol 5:305-322. CrossRef Medline

Stickney GS, Zeng FG, Litovsky R, Assmann P (2004) Cochlear implant speech recognition with speech maskers. J Acoust Soc Am 116:1081-1091. CrossRef Medline

Tokita J, Dunn C, Hansen MR (2014) Cochlear implantation and singlesided deafness. Curr Opin Otolaryngol Head Neck Surg 22:353-358. CrossRef Medline

van den Honert C, Stypulkowski PH (1987) Single fiber mapping of spatial excitation patterns in the electrically stimulated auditory nerve. Hear Res 29:195-206. CrossRef Medline

Vincent C, Arndt S, Firszt JB, Fraysse B, Kitterick PT, Papsin BC, Snik A, Van de Heyning P, Deguine O, Marx M (2015) Identification and evaluation of cochlear implant candidates with asymmetrical hearing loss. Audiol Neurootol 20 [Suppl 1]:87-89.

Wang X (2007) A sharper view from the top. Nat Neurosci 10:1509-1511. CrossRef Medline

Wang X, Kadner A, Scheich H (1999) Neuronal and behavioral discrimina- 
tion between upward and downward pulse interval modulation in cochlea implanted gerbils. Restor Neurol Neurosci 14:201-208. Medline

Wang X, Lu T, Snider RK, Liang L (2005) Sustained firing in auditory cortex evoked by preferred stimuli. Nature 435:341-346. CrossRef Medline

Watkin P, Baldwin M (2012) The longitudinal follow up of a universal neonatal hearing screen: the implications for confirming deafness in childhood. Int J Audiol 51:519-528. CrossRef Medline

Watkins PV, Barbour DL (2008) Specialized neuronal adaptation for preserving input sensitivity. Nat Neurosci 11:1259-1261. CrossRef Medline

Watkins PV, Barbour DL (2011a) Rate-level responses in awake marmoset auditory cortex. Hear Res 275:30-42. CrossRef Medline

Watkins PV, Barbour DL (2011b) Level-tuned neurons in primary auditory cortex adapt differently to loud versus soft sounds. Cereb Cortex 21: 178-190. CrossRef Medline
Weaver J (2015) Single-sided deafness: causes, and solutions, take many forms. Hearing J 68: 20:22-24.

Wilson BS, Dorman MF (2008) Cochlear implants: a remarkable past and a brilliant future. Hear Res 242:3-21. CrossRef Medline

Wilson BS, Dorman MF, Woldorff MG, Tucci DL (2011) Cochlear implants: matching the prosthesis to the brain and facilitating desired plastic changes in brain function. Prog Brain Res 194:117-129. CrossRef Medline

Zeng FG, Grant G, Niparko J, Galvin J, Shannon R, Opie J, Segel P (2002) Speech dynamic range and its effect on cochlear implant performance. J Acoust Soc Am 111:377-386. CrossRef Medline

Zhang F, Anderson J, Samy R, Houston L (2010) The adaptive pattern of the late auditory evoked potential elicited by repeated stimuli in cochlear implant users. Int J Audiol 49:277-285. CrossRef Medline 